\title{
Review
}

\section{Gamma Band Neural Stimulation in Humans and the Promise of a New Modality to Prevent and Treat Alzheimer's Disease}

\author{
Barry McDermott ${ }^{\mathrm{a}, *}$, Emily Porter ${ }^{\mathrm{a}}$, Diarmaid Hughes ${ }^{\mathrm{b}}$, Brian McGinley ${ }^{\mathrm{a}, \mathrm{c}}$, Mark Lang ${ }^{\mathrm{d}}$, \\ Martin O'Halloran ${ }^{\mathrm{a}}$ and Marggie Jones ${ }^{\mathrm{a}}$ \\ ${ }^{a}$ Translational Medical Device Lab, National University of Ireland Galway, Galway, Ireland \\ ${ }^{\mathrm{b}}$ College of Medicine, Nursing and Health Science, National University of Ireland Galway, Galway, Ireland \\ ${ }^{\mathrm{c}}$ Department of Computer Science \& Applied Physics, Galway-Mayo Institute of Technology, Galway, Ireland \\ ${ }^{\mathrm{d}}$ Centre for Astronomy, School of Physics, National University of Ireland Galway, Galway, Ireland
}

Handling Associate Editor: Amy Clements-Cortes

Accepted 2 July 2018

\begin{abstract}
Existing treatments for Alzheimer's disease (AD) have questionable efficacy with a need for research into new and more effective therapies to both treat and possibly prevent the condition. This review examines a novel therapeutic modality that shows promise for treating $\mathrm{AD}$ based on modulating neuronal activity in the gamma frequency band through external brain stimulation. The gamma frequency band is roughly defined as being between $30 \mathrm{~Hz}-100 \mathrm{~Hz}$, with the $40 \mathrm{~Hz}$ point being of particular significance. The epidemiology, diagnostics, existing pathological models, and related current treatment targets are initially briefly reviewed. Next, the concept of external simulation triggering brain activity in the gamma band with potential demonstration of benefit in $\mathrm{AD}$ is introduced with reference to a recent important study using a mouse model of the disease. The review then presents a selection of relevant studies that describe the neurophysiology involved in brain stimulation by external sources, followed by studies involving application of the modality to clinical scenarios. A table summarizing the results of clinical studies applied to AD patients is also reported and may aid future development of the modality. The use of a therapy based on modulation of gamma neuronal activity represents a novel non-invasive, non-pharmacological approach to $\mathrm{AD}$. Although use in clinical scenarios is still a relatively recent area of research, the technique shows good signs of efficacy and may represent an important option for treating $\mathrm{AD}$ in the future.
\end{abstract}

Keywords: $40 \mathrm{~Hz}$, Alzheimer's disease, gamma, neural stimulation

\section{INTRODUCTION: AN OVERVIEW OF ALZHEIMER'S DISEASE}

Dementia, characterized by cognitive impairment, affects between 24-50 million people globally [1,2].

\footnotetext{
${ }^{*}$ Correspondence to: Barry McDermott, Translational Medical Device Lab, 2nd Floor Lambe Translational Research Facility, University Hospital Galway H91 TK33, Ireland. E-mail: b.mcdermott3@nuigalway.ie.
}

This figure is expected to double every 20 years, until at least 2050 [2]. Alzheimer's disease (AD) is the most prevalent cause of dementia, accounting for about $70-80 \%$ of cases $[1,3]$. The key pathological, and perhaps defining, characteristic of $\mathrm{AD}$ is the presence in the brain of extracellular deposits of amyloid- $\beta(A \beta)$ and intracellular neurofibrillary tangles (NFT) of tau $[2,3]$. The typical cohort affected by $\mathrm{AD}$ are individuals aged over 60 years old, but it 
is increasingly being recognized that $\mathrm{AD}$ can affect younger people as well. Specifically, dementia rates in people under 50 years old are less than 1 in 4,000, of which $30 \%$ are due to AD [2]. Despite this, there is a clear correlation between onset and diagnosis of $\mathrm{AD}$ with age, with a higher prevalence in regions such as North America and Western Europe. These regions are also projected to have an increase in elderly population in the future, with a consequential increase in $\mathrm{AD}$ burden. Currently the annual cost of $\mathrm{AD}$ in the United States is of the order of $\$ 172$ billion [1].

$\mathrm{AD}$ is a complex multifactorial disease with an intricate, and as yet not fully understood, pathophysiology. Typically, the disease has a preclinical phase of decades. This early phase, if recognized, may be a key point for successful interventions [2]. Lifestyle factors such as diabetes, obesity, smoking, and depression have been highlighted as areas where modifications could decrease incidence of the disease, as is the area of vascular health [2].

Established AD in living patients has traditionally depended on clinical examination for diagnosis. Criteria such as the NINCDS/ARDA Alzheimer's criteria (National Institute of Neurological and Communicative Disorders and Stroke/The Alzheimer's Disease and Related Disorders Association) dating from 1984, are still in common use today with a sensitivity and specificity of $81 \%$ and $70 \%$, respectively for $\mathrm{AD}[4,5]$. It is increasingly accepted, however, as knowledge of $\mathrm{AD}$ grows that diagnosis is subtle and complex. While a definitive clinical diagnosis requires histopathological evidence by biopsy or autopsy, this is not usually feasible. However other diagnostic modalities, based for example on new knowledge of genetics and biomarkers, are becoming available which may result in more efficient detection of both established and early disease [6]. For example the apolipoprotein E4 (APOE4) gene is a major risk factor for $\mathrm{AD}$, with lifetime risk of $50 \%$ for homozygotes and 20-30\% for heterozygotes [2]. Biomarkers in the cerebrospinal fluid (CSF) such as $\mathrm{A} \beta_{42}$, total tau (t-tau), and phosphorylated tau (p-tau) offer a sensitivity of $85-90 \%$ in detecting $\mathrm{AD}$ in the prodromal stage [2]. Imaging modalities such as magnetic resonance imaging (MRI) and positron emission tomography (PET) also have significant diagnostic power [2, 7-9]. MRI offers structural information which may be harnessed with the knowledge that $\mathrm{AD}$ often causes neuronal loss in areas such as the hippocampus [2, 10]. PET modalities such as hexamethylpropylenamineoxime single emission computed tomography (SPECT) are used to differentiate between dementia types, while F-flurodeoxyglucose (FDG)-PET is sensitive to neural function and can be used to aid in AD diagnosis [2, 4]. Two new diagnostic techniques for $\mathrm{AD}$ that show promise are ocular and blood biomarkers. There is evidence that the retina nerve fiber layer at the rear of the eye thins in patients with both mild cognitive impairment (MCI) and $\mathrm{AD}$, detectable with optical coherence tomography [11]. Retinal A $\beta$ plaques also seem to be present in the eyes of AD patients and show correlation with brain $A \beta$ levels. Detection and quantification of the retina $A \beta$ levels could provide a non-invasive measure of AD [12]. Finally, blood biomarkers show diagnostic promise with evidence that $A \beta$ is rapidly transported from the CNS in the blood [13], and that measurement of blood based amyloid- $\beta$ protein precursor $(\mathrm{A} \beta \mathrm{PP})$ and composites may be correlated to brain $A \beta$ levels [14]. An aggregate of the results of several of these tests, including clinical examination, biomarkers, and imaging, may ultimately prove to have the best diagnostic power for AD [2].

While research and development of diagnostics for $A D$ has seen significant progress, treatment is a side of $\mathrm{AD}$ where there is an urgent need for new, more efficacious strategies. Current best treatment protocols can only result in a delay in the progression of the disease once established. Supportive care is the primary method of treatment, with maintenance of quality of life becoming difficult as $\mathrm{AD}$ advances $[6,15]$. There are only two classes of pharmacologic agents available for $\mathrm{AD}$ with both acetylcholinesterase inhibitors and N-methyl-Daspartate receptor antagonists showing only a modest slowing of the disease progression and alleviation of symptoms with more than half of all patients not responding at all [3]. Early intervention is becoming increasingly important in $\mathrm{AD}$, with one study concluding that elimination of modifiable risk factors, many related to vascular health, is capable of resulting in a $25-33 \%$ reduction in dementia [16]. As is often the case, a better understanding of the pathogenesis of the disease should result in more candidate therapeutic targets. In $\mathrm{AD}$, a large part of this pathogenic focus, and subsequent potential treatment strategies, has been at the molecular level $[3,17]$.

Therefore, this work provides a review of such a promising therapeutic avenue for $\mathrm{AD}$, based on increasing gamma activity in the brain. Gamma activity is electrical activity which occurs at frequencies ranging from $30-100 \mathrm{~Hz}$ [18]; with the $40 \mathrm{~Hz}$ gamma activity of most interest. Gamma activity may 
represent a novel, much needed, therapeutic target for $\mathrm{AD}$ prevention and treatment.

Prior to discussing gamma activity, the next section briefly reviews the current state of knowledge regarding the molecular pathophysiology of $\mathrm{AD}$ and candidate therapeutics deriving from these models, none of which have delivered adequate efficacy to date. Next, the third section introduces the concept of gamma neuronal activity through discussion of the findings reported in a recent key paper published by Iaccarino et al. [19]. The fourth section commences the review proper, examining papers of relevance from the early $1980 \mathrm{~s}$ to present day and is divided into subsections: the first focused on the underlying science, the second on the clinical application (with a table summarizing studies featuring application in AD), and a third on the potential limitations. Each paper is critically analyzed and summarized. Key findings are noted, with comparisons and contrasts to other papers discussed where relevant.

The review then concludes with a synopsis of the current state of the field and suggestions for future studies. To our knowledge, a work compiling and critically reviewing studies on gamma neuronal activity has not been conducted before. The proposed therapy based on gamma activity for AD is a unique nonpharmacological approach, showing signs of strong promise. The goal of this review is to consolidate and summarize the field in one coherent paper. The ultimate goal of research in this area would be the development and use of a device that modulates neural gamma activity in the brain, resulting in a way to treat and potentially even prevent AD.

\section{MOLECULAR PATHOPHYSIOLOGY AND THERAPEUTICS}

It is the intricately complex pathophysiology, particularly at the molecular and cell level, that makes AD difficult to tackle. Nonetheless, advances have been made in elucidating the mechanism of disease and with these advances comes the promise of novel therapeutics. Although the exact set of mechanisms and pathways behind $\mathrm{AD}$ are still unknown, a number of hypotheses on the pathology have been described in the literature which have resulted in associated candidate therapeutics.

The defining characteristic of $\mathrm{AD}$, the presence of $A \beta$ plaques and tau NFTs in the parenchyma and blood vessels of the brains of affected patients, has logically resulted in these two proteinaceous materials being at the core of hypotheses on the pathology of $\mathrm{AD}[2,3]$. One of the best known of these, the amyloid hypothesis, is nearly 30 years old. The amyloid hypothesis argues for accumulation of $\mathrm{A} \beta$ as the trigger, and even the driver, of AD. Deposition of $A \beta$ as plaques results in the downstream production of NFTs containing tau, with the overall course of the disease a result of an imbalance in the production and removal of $A \beta$ [20]. The evidence for the amyloid hypothesis stems largely from genetic studies. These studies demonstrate that mutations in the genes coding for $A \beta P P$ and presenilin peptides (PS1, PS2) result in abnormal accumulation of $\mathrm{A} \beta[2$, $3,20,21]$. A $\beta P P$ is a protein, metabolized by secretases to breakdown products. The presenilin peptides form catalytic subunits in $\gamma$-secretases, with mutations in these subunits resulting in less efficient A $\beta P P$ catabolism and the build-up in metabolites like the $\mathrm{A} \beta_{42}$ isoform. These $A \beta$ oligomers can then aggregate and form insoluble plaques, with changes in tau (hyperphosphorylation being of note) resulting in NFTs, a sequela of this deposition [2, 3, 20, 21]. Following on is a relatively simple linearly causal relationship between levels of $\mathrm{A} \beta$ plaques and tau NFTs in the affected brain and the resultant disease severity [2]. The amyloid hypothesis is schematically depicted in Fig. 1.

This hypothesis is being shown, as research continues, to be incomplete and to only partially explain the AD pathogenic puzzle. For example, tau has been shown to cause frontotemporal dementia without the presence of $A \beta$ plaques, thereby working independently [2]. Further, the amyloid hypothesis explicitly predicts that other causes of $\mathrm{AD}$ would relate to the $\mathrm{A} \beta$ production and clearance balance [21]. An important risk factor for $\mathrm{AD}$, APOE4, has been shown to contribute to $\mathrm{AD}$ in a variety of ways including modulation of the $A \beta$ deposition and removal balance but also by other mechanisms independent of $A \beta$. These independent mechanisms include, for example, impairment of defensive systems, deregulation of neuronal signaling, and impairment of interneuron function [22]. Finally, a fundamental concern about the amyloid hypothesis is the fact that the normal function of $A \beta P P$ or $A \beta$ is largely unknown [21]. Thus, it is unlikely that this hypothesis could explain the full pathogenesis with such a knowledge gap concerning two of the major players [10]. Indeed current opinion seems to indicate that it is possibly tau and not $A \beta$ that may be the main driver behind $\mathrm{AD}$ as a standalone "tau hypothesis" $[23,24]$. 


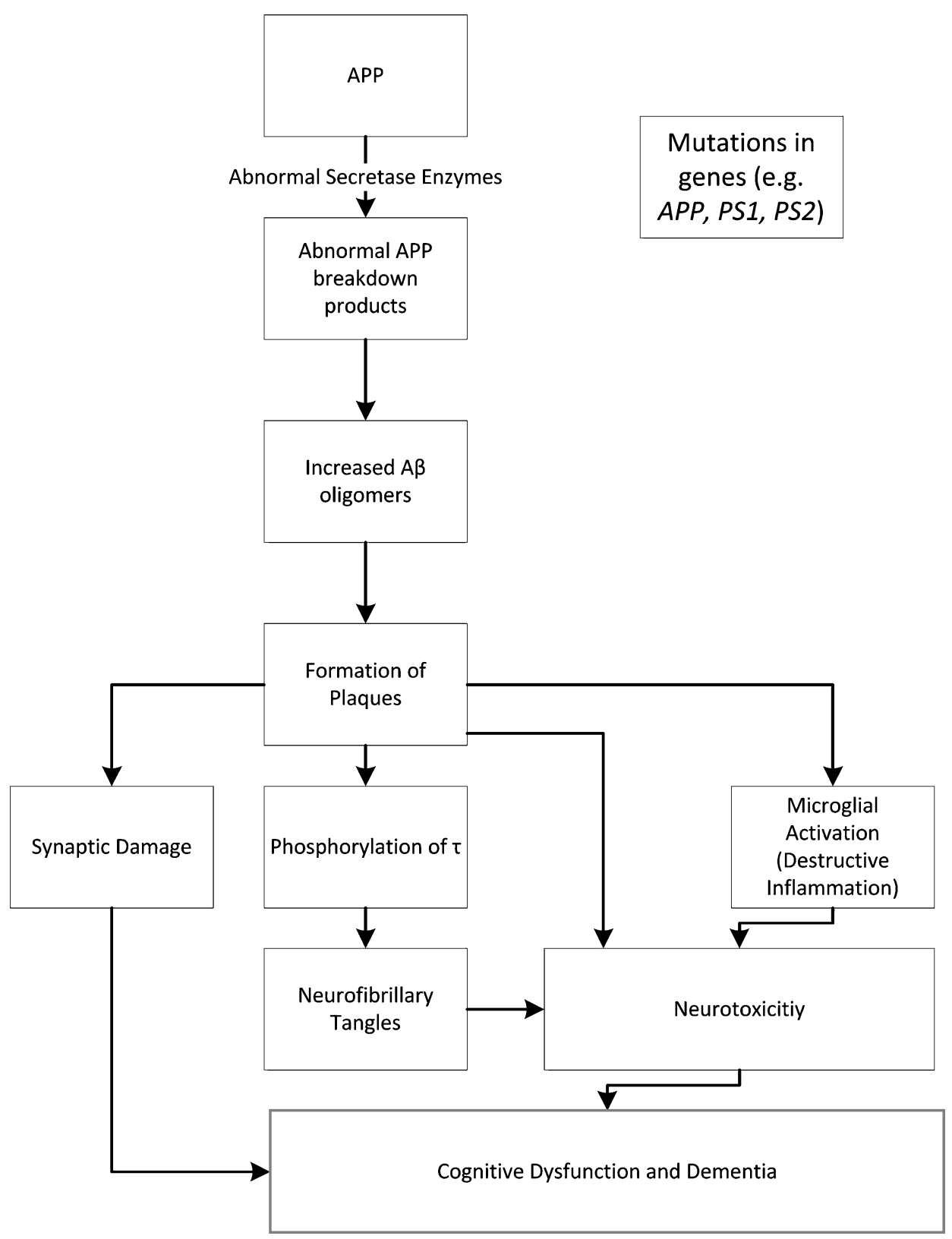

Fig. 1. The Amyloid Hypothesis (adapted from [3, 20, 21]).

Despite these gaps and uncertainties, it is logical to assume, given the ubiquitous presence of $A \beta$ and tau in affected patients, that targeting $\mathrm{A} \beta$ production, removal, or other associated biochemical pathways should prevent or treat $\mathrm{AD}$, as should similar approaches directed at tau. The anti-amyloid approach targets many points in the A $\beta P P$ metabolic pathway, for example modulating the secretase enzymes, preventing $A \beta$ aggregation, enhancing enzymes that degrade $A \beta$ oligomers, and promoting an immune response to the presence of $A \beta$ through the use of vaccines [3, 17]. With regards to tau, agents blocking hyperphosphorylation as well as targeting later points in the pathway such as inhibiting oligomerization and enhancing degradation are the subject of active research [3, 17]. However, to date these approaches have shown disappointing results [2]. Immunotherapy may offer the most promise of therapeutic efficacy, with both active and passive therapies targeting $A \beta$ in clinical trials [25]. Of these, 
passive immunotherapy with monoclonal antibody agents have performed best to date, with candidate molecules like aducanumab showing efficacy but also significant safety issues [26].

Other AD hypotheses have emerged that complement and add to the original amyloid hypothesis with the aim of more thoroughly describing the disease pathway, and hopefully highlighting novel therapeutic targets. These include, for example, the inflammatory hypothesis, cholinergic hypothesis, metal hypothesis, a hypothesis proposing a fungal etiology [27-30], and also a possible link between AD and diabetes involving brain insulin resistance [31, 32]. Indeed the only two classes of drug, comprising four agents, currently in use for direct treatment of the symptomatic phase of $\mathrm{AD}$ are based on cholinergic, and closely related, targets $[27,33]$.

Overall, however, the amyloid hypothesis of $\mathrm{AD}$ along with the closely related tau hypothesis remain the core models describing the molecular pathogenesis of the disease. The models offer the most promise, despite being incomplete [21], with other hypotheses being developed to complement and attempt to fully explain the pathway of events on the molecular and cell level. With these models come logical therapeutic targets at points along the pathways. However, to date pharmacologic agents acting on these targets have failed to produce the efficacy desired or expected by the models of disease [27-29, 33, 34]. The implication is that the models are as yet imperfect. The reality is that despite it being nearly 30 years since the development of the amyloid hypothesis [20], effective treatments for $\mathrm{AD}$ are still not in existence, with the epidemiology of the disease making the development of such treatments an absolute necessity for society [1]. Despite this disappointment, it is hard to move away from the central pathognomonic feature which is the presence of $A \beta$ plaques and tau NFTs. Even with the disappointing results to date, it is still rational, based on the wide body of evidence, to consider that a novel technique targeting this point in the pathology should give solid clinical improvement in AD patients.

\section{GAMMA BAND NEURAL STIMULATION: A NEW PREVENTATIVE AND THERAPEUTIC HOPE}

A novel, non-pharmacological approach to AD and other neurological pathologies involves manipulating gamma activity in the brain. Gamma electrical

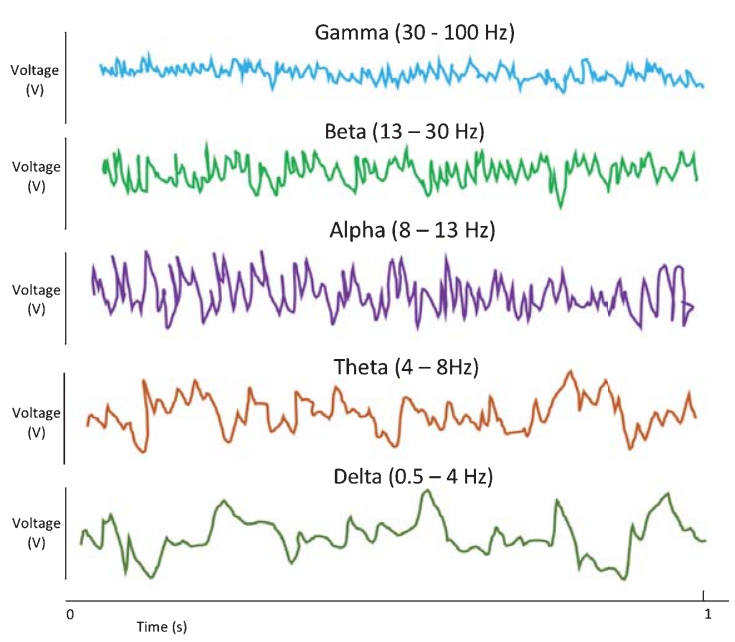

Fig. 2. EEG neural oscillatory patterns. These patterns may be divided into groups based on frequency range, with gamma activity being the highest frequency grouping. The frequencies ranges listed are approximate $[18,35]$.

activity refers to electroencephalogram (EEG) oscillations at a frequency of approximately $30-100 \mathrm{~Hz}$ in localized central neural pathways. This electrical activity has been related to many sensory and cognitive functions [18]. Gamma electrical oscillations are one group of oscillation patterns seen on EEG, with the others (delta, theta, alpha, and beta) being of lower frequency activity [35]. These are illustrated in the sketch in Fig. 2.

The power of gamma activity is increased during the processing of sensory information and in cognitive tasks that involve memory [36]. Further, the increase in gamma activity seen in these tasks is also associated with decreases in the power of the other, lower frequency patterns (delta, theta, alpha, and beta) [36]. Finally, AD patients may feature a reduction in the power of gamma activity [36, 37]. Therefore, modifying gamma activity for patients with AD may support improved cognitive function.

An important set of studies demonstrating the link between gamma activity and $\mathrm{AD}$ was published by Iaccarino et al. using primarily the 5XFAD mouse model of AD [19]. An important cell type of interest in the study was that of interneurons, that diverse collection of neural cells that vary in morphology, connectivity, and physiology [38]. The results of [19] demonstrated reduced levels of the power of gamma activity in the hippocampus of the 5XFAD mice relative to the wild type. Further, this reduction in gamma activity was observed before the accumulation of amyloid plaque or evidence of cognitive impairment. 
It was further shown that increasing gamma activity in the CA1 region of the hippocampus, by stimulation of optogenetically modified interneurons using blue light at $40 \mathrm{~Hz}$, reduced $A \beta$ levels by approximately $50 \%$ and was mediated by both neural and microglia mechanisms. The neural response was evidenced by reduced $A \beta P P$ degradation as well as modifications to endosomal activity. Microglia activity was increased with both genetic evidence of upregulation in the microglia of genes related to phagocytosis, and histological evidence of microglia adopting phagocytotic morphology, increasing in numbers in the area under study and an increase in the number of microglia with internalized $A \beta$. These effects were also observed in the visual cortex, with $40 \mathrm{~Hz}$ white light stimulus supplied as an externally flickering source observed visually by the mice. Importantly, the external supply of the stimulus, delivered via the retina, optic nerve and subsequent pathways was translated as an increase in gamma activity in the visual cortex. Also, of interest was the discovery that GABAergic neurons are likely involved in the process, with the use of a GABA antagonist nullifying the neural and microglial mediate effects. The protective effects of simulation of gamma activity were found to both prevent $A \beta$ production and to reduce established plaques following daily 1-hour exposures to the flickering stimulus over a week. Of note is that fact the effect on soluble $A \beta$ was transient with 1 hour of stimulus exerting effects lasting between 12-24 hours, hence the need for daily stimulation, with a resultant cumulative effect over a week in order to tackle established plaques. This study provided compelling evidence suggesting that stimulation of gamma activity may offer a novel therapeutic model for AD. Further the effects were also demonstrated in an extension of the study to another mouse model of $\mathrm{AD}$, the TauP301S model, with a similar microglia response and, in this model, a reduction in p-tau observed [19]. Significantly, these effects were seen only with a $40 \mathrm{~Hz}$ stimulus, and not with $20 \mathrm{~Hz}, 80 \mathrm{~Hz}$ or random frequencies.

While this study made significant strides toward demonstrating the role of gamma activity in $\mathrm{AD}$, an examination of behavioral endpoints was not considered. Evidence of cognitive improvement in the mice following stimulation of gamma activity would lend more weight to the hypothesis of gamma activity as an AD treatment. Similarly, it would be of interest to investigate if external stimulation of gamma activity results in an increase in endogenous gamma activity. Furthermore, while the authors of [19] mention the transient effect of the $40 \mathrm{~Hz}$ flicker, it may be conceivable that repeated, long term treatment may result in some recovery of normal gamma activity in affected individuals, given the plasticity of the brain.

This paradigm of external stimulation, evoking gamma activity in the brain, particularly at $40 \mathrm{~Hz}$, is the focus of this review. While it is accepted that there is no guarantee that therapy based around gamma activity modulation is the much-needed miracle treatment for $\mathrm{AD}$, it is one of the more promising avenues of research and worthy of further studies.

\section{REVIEW OF GAMMA BAND STIMULATORY AND RELATED STUDIES}

In this section, a representative review of the field is performed, exploring the reported studies over the past decades up to the present day. Subsections examine both the underlying neurophysiology involved in external stimulation as well as the possible clinical application (with emphasis on AD) of using the phenomenon of gamma activity as a protective and therapeutic tool in AD patients. Each subsection features an analysis of the findings from relevant papers to explore and explain the area under examination in the section, with the studies presented in chronological order. Although the focus of the review is on gamma activity, defined as neural oscillations in the $30-100 \mathrm{~Hz}$ range [18] and in particular the discrete $40 \mathrm{~Hz}$ point, some of the material discussed is not in this band but is included as it is relevant to the overall discussion and narrative of the field. Throughout this section, information reported from the studies is described using the past tense whereas our commentary uses the present tense. A final subsection discusses potential limitations of using external stimulation in a clinical context.

\section{Neurophysiological basis of brain stimulation by external sources}

Brain electrical potentials can be elicited in response to a triggering event, with the trigger being either endogenous (for example a thought), exogenous (external stimuli), or indeed a mixture of the two [39]. Further, these event-related potentials (ERPs) can be classified as evoked, where the ERP is phase locked to the stimulus, or induced where the ERP is not phase locked [40, 41]. The ERPs can be recorded using technologies such as EEG or magnetoencephalography (MEG). In EEG, the electrical potentials due to neuronal activity are recorded using 
electrodes, typically placed on the scalp. Information such as electrical power with respect to time and frequency, as well as the topological pattern of activity, may be derived from the recordings [42]. MEG is a similar modality which records the magnetic fields produced by the electrical activity using magnetometers, resulting in the derivation of similar information [42].

With regards to external stimuli, a range of modalities including visual, auditory, and somatosensory are found in the literature with exact implementation of each varying depending on the study. These modalities of stimulation and the nature of the ERPs produced gives insight in to the mechanisms involved in neural stimulation and it is about this physiology that the subsequent papers reviewed give an understanding. Any proposed therapeutic modality based on external stimulation can only realistically be effectively implemented with sufficient knowledge of the normal pattern of responses that result from such stimuli. Hence, in the subsequent three subsections, the studies are divided roughly in terms of the primary stimulatory modality used; auditory, visual, and somatosensory. This division is somewhat artificial, but necessary to coherently present the studies. After these initial three, the subsequent subsections deal with studies concerning a variety of ancillary but important information relating to the field.

\section{Event related potentials from auditory stimuli}

The initial stimulatory modality reviewed is that of auditory stimuli. It is useful to clarify the precise meaning of the terms "monaural" and "binaural" when used alone or when referring to auditory stimulation in the form of beats. Monaural refers to stimulation of one ear; while binaural involves stimulation of both ears. However, when in relation to beats (i.e., the interference pattern resulting from two sound waves of nearby frequencies), the phrase 'monaural beats' indicates that the two sound waves are presented to both ears simultaneously, while 'binaural beats' means the interfering sound waves are presented to both ears separately.

As shall be described in the sample papers reviewed below, auditory stimuli vary from clicks to bursts and from beats to pulses. Further, they can be delivered to individuals in a variety of ways including through monaural and binaural stimulation and at a range of frequencies and intensities. Despite this variety, there is a coherence in the protocols used and results obtained which allows the derivation of valuable insights into the nature of ERPs and brain activity.

Galambos et al. in 1981, reported on brain potentials elicited in response to auditory stimuli [43]. These ERPs contained a subset of waves appearing 8-80 $\mathrm{ms}$ after the stimulus called the middle-latency response (MLR). Brain potentials were recorded between two electrodes, one at the forehead and another on the earlobe of the stimulated ear. The auditory stimulus was comprised of monaural clicks or tone bursts delivered via an ear phone. The clicks were delivered at $10 \mathrm{~Hz}$ while the tone bursts consisted of a $500 \mathrm{~Hz}$ tone supplied at different frequencies including $40 \mathrm{~Hz}$ lasting $6 \mathrm{~ms}$ with a $2 \mathrm{~ms}$ rise and fall. It was found that MLRs were generated in response to the auditory input took the form of 3 or 4 cycles of a $40 \mathrm{~Hz}$ sine wave. Superposition of waves generated from successive stimuli occurred with constructive interference in the form of maximal amplitude, most evident, if the stimulus was supplied at a rate of $40 \mathrm{~Hz}$. The amplitude of the MLR response was also found to increase in response to the amplitude of the stimulus, although the lag between stimulus and resultant MLR was also seen to increase.

Another interesting phenomenon discovered in [43] was that a drop in the frequency of the stimulus resulted in an amplitude rise and latency increase. This latter observation was ascribed to the physiology and anatomy of the inner ear, with a lower frequency causing more of the basilar membrane of the cochlea to be recruited and hence more neurons to be stimulated which takes a longer time but would result in a stronger amplitude of transmitted signal. The MLR was reflective of activity in the auditory pathway from ear to brain [43]. Further, it seemed that most of the MLR was generated from the cortex as most of the response was generated from the forehead electrode. The paper also noted that a corresponding $40 \mathrm{~Hz}$ response was seen in response to both visual and olfactory stimuli as well as other less obvious stimuli such as performing cognitive tasks. The study hence provides evidence that this $40 \mathrm{~Hz}$ rhythm may correspond to a state of 'cortical arousal' or be needed for processing of sensory and other information. Controls were used to rule out electrical or physiological artefacts as a source of these effects. It is of interest that the resultant $40 \mathrm{~Hz}$ neural response occurred regardless of the stimulus frequencies used in this study. Also, the gamma activity featured superposition, as evidenced by constructive interference and maximal amplitude when the stimulus was delivered at $40 \mathrm{~Hz}$. 
ERPs to auditory stimuli were the subject of a 1996 study by Pantev et al. [44]. The MLR waves, studied previously by Galambos et al. [43], was the subject of examination along with slow response waves, occurring 40-250 ms after stimulus. The MLR can be divided into component parts when recorded on EEG or MEG [45]. These components have characteristic shapes of deflection and timing of latency [45]. For example, the first vertex negative wave of the MLR is referred to as the Na response on EEG (Nam on MEG), the Pa component (Pam on MEG) relates to a positive deflection arising from the Heschl's gyrus (the location of the primary auditory cortex), and the N1 (N1m on MEG) component is a strong negative component with a characteristic latency of about $100 \mathrm{~ms}$ [45]. In [44], it was in particular the Pam component of the MLR and the $\mathrm{N} 1 \mathrm{~m}$ component of the slow response that were the responses under examination. The so called steadystate response (SSR), and a corresponding steady state field (SSF) if using magnetic recordings, refer to the maximal potential or field invoked by stimuli occurring close enough together to allow superposition of the ERP. In this study it was the SSF that was recorded, as opposed to the potentials recorded by the comparative work of Galambos [43]. In the Pantev study, participants were exposed to Gaussian tone pulses at a repetition rate of $39 \mathrm{~Hz}$ (which the authors referred to as the ' $40 \mathrm{~Hz}$ ' steady state stimulation) with a half time of $5 \mathrm{~ms}$ and carrier frequencies of $250 \mathrm{~Hz}, 500 \mathrm{~Hz}, 1000 \mathrm{~Hz}, 2000 \mathrm{~Hz}$, and $4000 \mathrm{~Hz}$. Each stimulus was of $200 \mathrm{~s}$ duration and used twice in random order at $60 \mathrm{~dB}$ intensity, administered using magnetically silent means through a silicon ear piece into the right ear. A 37 channel biomagnetometer sensor array was positioned over the left temporal lobe (location of an auditory cortex) to record the MEG resulting from the stimulation. The source location of the SSF was calculated for each carrier frequency and the corresponding anatomical landmark extrapolated using MRI. The study found a maximal field intensity in response to the $250 \mathrm{~Hz}$ carrier, with the lowest for the $4000 \mathrm{~Hz}$ carrier. This difference in amplitude response was suggestive of different response source generator sites at the different carrier frequencies. These source locations for all SSF responses were in the auditory cortex as expected but of key interest in the study was the finding that the precise location showed a medial shift as frequency increased characteristic of $\mathrm{N} 1 \mathrm{~m}$ as opposed to a lateral shift that would be expected of Pam. The source location for both Pam and SSF were similar when a $500 \mathrm{~Hz}$ tone was used, in agreement with the study of Galambos [43], but the Pam and SSF sources showed divergence with changing frequency, the SSF following that medial pattern of the N1m sources and not the lateral pattern of Pam sources. This finding implied that the $40 \mathrm{~Hz}$ SSF was not comprised of summated MLRs as postulated by Galambos, but rather summation of the slow response waves that occurred later after the stimulus. In [44], it was proposed that this effect was due to a nonlinearity in neural assemblies and a frequency dependence effect.

This study added to the work of Galambos, with the key additional finding that it is the later, slow response waves, that may be responsible for the SSR or corresponding SSF. The movement in source location within the auditory cortex with frequency is of particular interest. This spatial response perhaps was explained by differing regions of the cochlea being stimulated depending on the stimulus rates with the resultant impulse distributing to differing tonotopic arrangement of neurons. Also, of note was an amplitude response with lower frequency carrier waves resulting in maximum field intensity of the ERP.

Ross et al. studied the SSR produced in response to sinusoidal amplitude modulated (AM) tones using MEG to record the responses in healthy participants [46]. Various carrier frequencies were separately modulated by 30 different frequencies ranging from 10-98 Hz. These 30 different stimuli were delivered monaurally to 8 participants, with each stimulus lasting $200 \mathrm{~s}$ in duration. The SSR was recorded using MEG as a spectrum composed of components with distinct amplitude and phase. It was found that each SSR produced (and hence the elicited cortical activity) matched the corresponding modulation frequency, with activity also at harmonics. Interestingly, modulation frequencies that were a harmonic of, or near, $40 \mathrm{~Hz}$ had a clear and often dominant spectral peak at the $40 \mathrm{~Hz}$ point. For example, the spectrum resulting from $10 \mathrm{~Hz}$ and then $14 \mathrm{~Hz}$ had predominant $40 \mathrm{~Hz}$ and $42 \mathrm{~Hz}$ components respectively, while the $40 \mathrm{~Hz}$ spectrum was dominated by the fundamental peak at $40 \mathrm{~Hz}$.

Pastor et al. investigated click based auditory stimulation at a variety of frequencies in the gamma range (including $40 \mathrm{~Hz}$ ) using an ear phone positioned in the right auditory canal of 28 participants [47]. The evoked SSR was analyzed using both EEG and PET. With regards to EEG, 21 electrodes were arranged according to the 10-20 system and 500 responses (in $500 \mathrm{~ms}$ epochs) were averaged for each stimulation frequency. The power spectrum was calculated for all 
stimulation frequencies with the dominant frequency and also the $40 \mathrm{~Hz}$ response analyzed. It was found that maximal SSRs were recorded at the F3 electrode and the responses here were compared to the results from PET. The PET part of the study consisted of a similar experimental protocol, with 9 participants from the original group with EEG results representative of the entire group used. Four distinct frequencies $(12 \mathrm{~Hz}, 32 \mathrm{~Hz}, 40 \mathrm{~Hz}$, and $47 \mathrm{~Hz})$ were presented as clicks, with the responses captured over the course of a 20-minute scan, one scan for each stimulation frequency. The basis of the PET scan was the measurement of a proxy for synaptic activity, regional cerebral blood flow (rCBF).

The results from the EEG part of the study found that the SSR oscillated at the same frequency as the stimulus (which correlates with the results of Hermann et al. which studied visual stimuli [48]) but that the greatest amplitude was reached when using a stimulation frequency of $40 \mathrm{~Hz}$. Interestingly, the largest responses were found to occur at the F3 electrode, which was the side contralateral to the stimulated side. The use of PET allowed the study of responses from a greater range of brain structures than had been the case in previous studies, which focused on the cortex and thalamus. It was found that the stimuli in the gamma frequency range caused a change in $\mathrm{rCBF}$ in the auditory areas of the brain in a pattern similar to that observed using EEG. The greatest effect was again seen at $40 \mathrm{~Hz}$ stimulation frequency with a contralateral response pattern confirmed by an increased $\mathrm{rCBF}$ in those areas at all stimulation frequencies. The contralateral primary auditory cortex was activated but also of note was a second smaller area surrounding the primary auditory cortex which may have a special role in temporal auditory pattern detection.

In addition, it was found that $40 \mathrm{~Hz}$ stimulation uniquely caused an increase in $\mathrm{rCBF}$ in the cerebellum, particularly on the side contralateral to the stimulus. This area of the brain has an important role in processing of auditory information. The authors of [47] postulated that the cerebellum only becomes more active at some resonant stimulation frequencies, particularly $40 \mathrm{~Hz}$. Although the cerebellum is noted by [47] to have a role in auditory processing, this cerebellar activation was only seen at $40 \mathrm{~Hz}$ stimulation and demonstrates that this 'special' frequency activates areas of the brain outside of those classically linked to a given stimulus modality.

This study also links the SSR to an increase in cortical synaptic activity in addition to the influence of superposition of MLR potentials (as proposed by Galambos [43]) and phase synchronization of pools of cortical neurons (which could be interpreted as the neuroanatomical explanation of Hermann [48]). The patterns of increases in $\mathrm{rCBF}$ in the auditory cortex observed on PET in response to the stimuli suggests this is the case since rCBF is directly correlated to neuronal synaptic activity.

A 2004 study by Artieda et al. investigated SSRs produced in response to auditory stimulation in the form of a $1200 \mathrm{~Hz}$ tone amplitude modulated by a sinusoid of linearly increasing frequency, ranging from $1-120 \mathrm{~Hz}$ (a so-called linear "chirp") [49]. The purpose of this form of modulation was to allow the simultaneous and rapid examination of SSRs evoked from a range of different frequencies as opposed to evoking individual frequencies in separate experiments. 10 participants were exposed to the stimulus which was delivered binaurally with the sound lasting $1.61 \mathrm{~s}$. The response was measured using EEG. A minimum of 500 sweeps was recorded for each subject with the average SSR calculated. It was found that the frequency of the SSR matched that of the stimulus with two maximal response points observed, the first around $45 \mathrm{~Hz}(30-60 \mathrm{~Hz})$ and a smaller one at $80-120 \mathrm{~Hz}$. The maxima around $40 \mathrm{~Hz}$ was postulated to be originating in the auditory cortex with some contribution from the brain-stem. The smaller maxima was thought to likely originate in the brainstem.

The explanation proposed in [49] for the maxima near $40 \mathrm{~Hz}$ was due to phase-locking and a higher level of synaptic activity at this stimulation frequency. Further, it is suggested that $40 \mathrm{~Hz}$ is one of the "working" frequencies of the brain. Interestingly, it was observed that the cerebellum is also activated at a stimulation frequency of $40 \mathrm{~Hz}$ and may act as a brake on the extension of the activity. Finally, in [49] the SSR was analyzed in two sleeping participants with the effect present but with a lower amplitude.

The effect of sound stimulation, as monaural or binaural beats, on neural electrical activity, was the basis of a 2015 study by Becher et al. [50]. The effect on neural electrical activity was measured using intracranial EEG, with EEG data analyzed for five distinct channel groups (for example a mediotemporal depth location and a surface location). The metrics derived from EEG included power and phase synchronization, with the latter referring to an increased stability of phase relationships between different brain regions as measured as the phase differences between all possible channel pairs within 
the 5 channel groups [50]. The beat stimuli were produced as amplitude-modulated signals. Monaural beats were produced by superimposing two sine waves of similar frequencies. For example, a $460 \mathrm{~Hz}$ carrier wave with $40 \mathrm{~Hz}$ modulation was produced by superimposing $440 \mathrm{~Hz}$ and $480 \mathrm{~Hz}$ sine waves. This signal was then presented simultaneously to both ears. In the case of binaural beats, both sine waves were presented separately to both ears with the perception of a $40 \mathrm{~Hz}$ modulated signal generated as a result of the body's own sound location mechanism [50,51]. Beat frequencies of $5 \mathrm{~Hz}, 10 \mathrm{~Hz}$, $40 \mathrm{~Hz}$, and $80 \mathrm{~Hz}$ were used as stimuli with the nonsuperimposed waves used as controls. These were presented to 10 temporal lobe pre-surgical epilepsy patients as $5 \mathrm{~s}$ stimulations with intervals in between. The response of the patients was recorded as EEG signals from electrodes which varied in position, number and placement between patients but included hippocampal depth electrodes, strip electrodes on the surface of the temporal lobes and surface electrodes. The effect of the stimuli was studied as the effect on the power and phase synchronization of the resultant EEG signals, with a wide variety of diverse results produced depending on the nature of the stimulus. It was noted in [50] that it was unclear why certain beat stimuli produced significant effects in a particular direction and others did not affect the EEG signal to any great extent. In most cases the result found was a decrease in EEG power and synchronization, most significantly with a $5 \mathrm{~Hz}$ monaural beat frequency and $80 \mathrm{~Hz}$ binaural beat frequency. Of interest was the effect seen with a $40 \mathrm{~Hz}$ monaural beat frequency which caused the most pronounced increase in power. This result was in agreement with other studies which hypothesize that interneuron networks are most responsive to $40 \mathrm{~Hz}$ stimulation [52]. Interestingly, these interneuron networks are thought to play a role in sound processing through effects on pyramidal cells [50, 53].

As discussed in the section on the molecular pathophysiology of $\mathrm{AD}$, pyramidal cell activity is a key part of the cholinergic hypothesis [27]. Phase synchronization is also thought to be important in cognition and memory, including synchronization of gamma activity [50, 54]. It was suggested in [50] that a stimulus that results in phase synchronization could be of significance in applications involving cognition. Further, it is known that several conditions including AD, along with epilepsy and Parkinson's disease, may feature and be connected to abnormal synchronization $[50,55]$. In the particular set of stimuli used in [50], a
$5 \mathrm{~Hz}$ binaural beat frequency were found to increase phase synchronization with, disappointingly, a $40 \mathrm{~Hz}$ monaural beat frequency found to decrease synchronization. It would also have been of interest to use a $40 \mathrm{~Hz}$ signal as a control. Nevertheless, auditory stimulation in the form of beats is yet another example of a variant of the auditory modality that can modulate neural activity and may offer therapeutic effects through these modulatory effects. Of note, it is mentioned in [50] that the surface response specifically at the $40 \mathrm{~Hz}$ point may be a response to prolonged auditory stimulation, not specifically beats, whereas other frequency responses were specific for beats.

As well as different stimulation modalities, within a given modality there is variation available. This is reflected in the different types of auditory stimulation used in the studies discussed above. Importantly, a specific type of stimulation will have different properties and can result in different responses. This area was the subject of a 2016 study by Voicikas et al. investigating the nature of $40 \mathrm{~Hz}$ SSRs generated in response to two different types of auditory stimuli, clicks and flutter amplitude modulated tones (FAMs) [56].

A FAM tone differs to that of a regular AM tone. In an AM tone, the amplitude of a carrier wave is varied by that of a lower frequency messenger signal. The mathematical description of a simple sinusoidal AM tone is given by equation 1 , where $f_{c}$ is the carrier frequency, $f_{m}$ is the messenger frequency and $\mathrm{s}(\mathrm{t})$ is the amplitude of the AM signal over time $t$. The corresponding equation for a FAM is then given by Equation 2. The waveforms of both types of tone (AM and FAM) over a $0.05 \mathrm{~s}$ interval with a $f_{c}$ of $440 \mathrm{~Hz}$ and a $f_{m}$ of $40 \mathrm{~Hz}$ are shown in Fig. 3. FAMs may be considered a form of isochronic tone, where a single tone is presented as pulses with even spacing between the pulses [57]. FAMs feature the same length of sound and pause, whereas AM tones have no pause phase.

$s(t)=\sin \left(2 \pi f_{c} t\right) \sin \left(2 \pi f_{m} t\right)$

$\mathrm{s}(\mathrm{t})=\left\{\begin{array}{cc}\sin \left(2 \pi f_{c} t\right) \sin \left(2 \pi f_{m} t\right) & \sin \left(2 \pi f_{m} t\right)>0 \\ 0 & \text { otherwise }\end{array}\right.$

Clicks are a popular type of auditory stimulus, producing large reliable responses [56, 58, 59], and are commonly used to generate a response in gamma activity in clinical cohorts $[56,60]$. Click stimuli have steep rise and fall times, long pauses between sounds, 

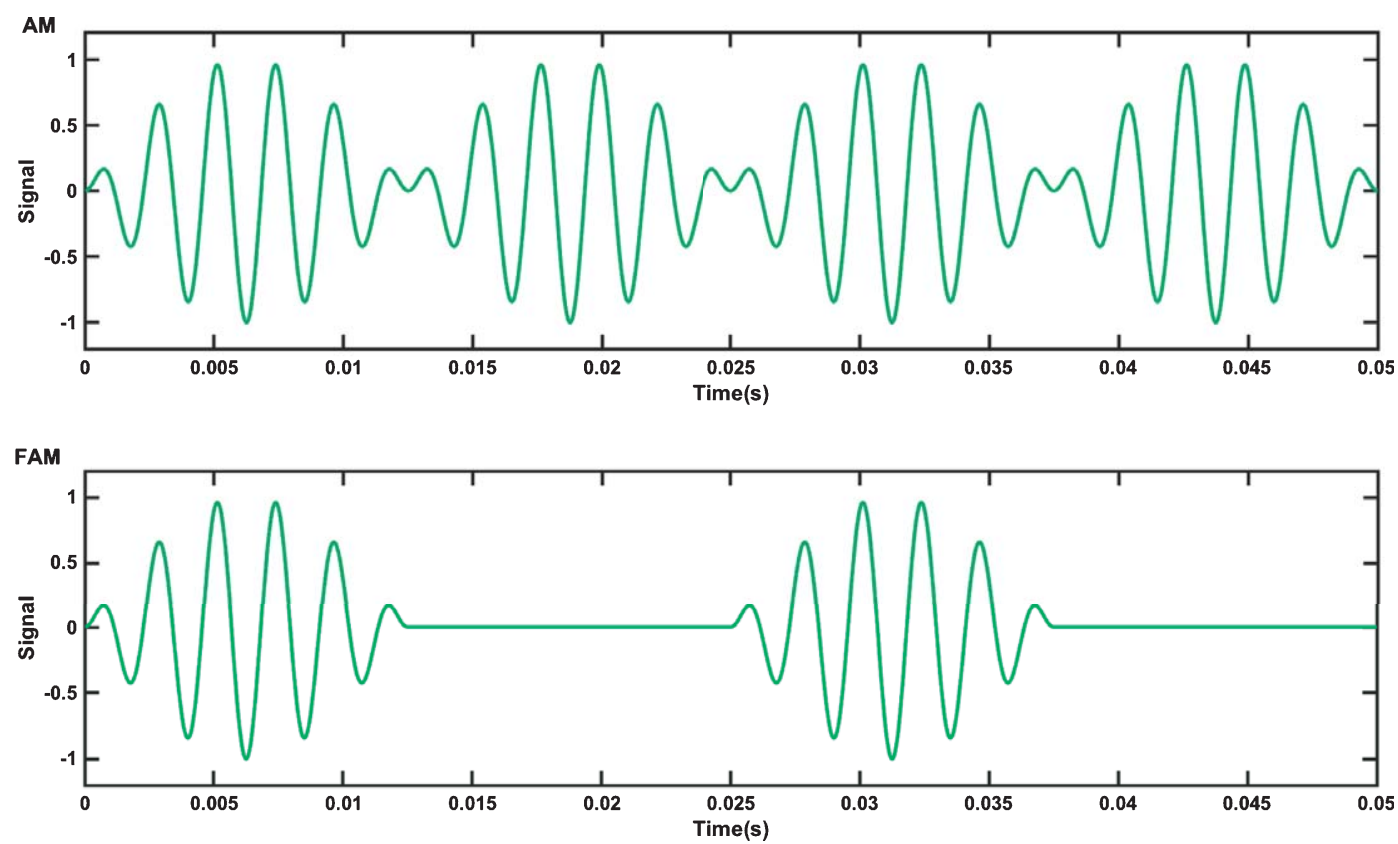

Fig. 3. Top: Amplitude modulated (AM) tone and Bottom: Flutter amplitude modulated tone (FAM) waveforms.

and contain low and high frequency components resulting in wide activation of the auditory cortex [56, 61, 62]. FAM stimuli also feature periods of silence and sound with sharp steep rise and fall times but smoother transitions as compared to clicks [56]. The study of Voicikas in particular was interested in assessing how the SSRs generated in response to these two different auditory stimuli were affected by concurrent tasks being done by participants and also the subjective perception of the stimuli by the participants [56]. As reported in [43], the SSR tends to the same frequency as the external stimulus with greatest effect seen at $40 \mathrm{~Hz}$. The FAM stimulus used in [56] was generated using a $440 \mathrm{~Hz}$ carrier wave with $40 \mathrm{~Hz}$ amplitude modulation. Half of a $25 \mathrm{~ms}$ cycle was tone, with the other half silence. The click stimulus consisted of bursts of white noise $1.5 \mathrm{~ms}$ in duration. The stimuli were presented binaurally to 30 participants with the SSR recorded using EEG. Both stimuli resulted in SSRs of $40 \mathrm{~Hz}$ as expected with the click stimulus found to result in a different pattern of response topology on EEG compared to the FAM stimulus; more central and wide for clicks and more frontal for FAM. This was thought to be a result of the different frequency profiles of the two stimuli types resulting in activation of different parts of the cortex [56].

In the initial part of the study, a subjective assessment of each stimulus in terms of arousal and pleasantness was conducted in a different (male only) cohort to the main part of the study. FAMs were found to be less arousing but more pleasant [56]. In the next part of the study, the effect on the SSR due to concurrent tasks (stimuli counting, reading and resting with closed eyes) was assessed. This part was used to assess the effect of changes in attention on the SSR. It was found that SSRs due to FAM stimulus was not affected by the concurrent tasks whereas SSRs to click stimulus were. The conclusion of [56] was that FAM auditory stimulation may be more suitable than clicks in clinical settings, where control of attention may be difficult. A related study by Griskova-Bulanova et al. also concluded that FAM stimulation may be more suitable than clicks when applied specifically to schizophrenic patients with also the resultant SSR from the FAM stimulation having the potential to be used as a biomarker for the condition [63]. A final observation from the results of [56] is the different topological response patterns resulting from the two stimuli type. These response patterns show that as well as causing an SSR within the auditory cortex, different parts of the cortex may be targeted depending on the nature of the stimulus used. Also of note is the production of a $40 \mathrm{~Hz}$ SSR to an AM stimulus that uses a $40 \mathrm{~Hz}$ modulating frequency, indeed $[50,52]$ indicate that the SSR in general will occur at the same frequency as the modulating frequency. 
To summarize the results of this subsection, the seven relevant studies focusing on auditory stimulation give a good introductory insight as to the nature of ERPs and the inherent complexity and subtleties therein. ERPs are generated in response to a range of auditory stimuli and can be readily detected and recorded. The ERPs feature superposition, resulting in the creation of a SSR (or SSF) [43, 44]. The precise neural activity that comprises the ERPs and consequent SSRs is complex due to a proposed nonlinearity and frequency dependence effect [44] and may be due to the MLR waves appearing $8-80 \mathrm{~ms}$ after the stimulus [43], or later slow response waves that appear 40-250 ms after the stimulus [44]. An increase in cortical activity may also contribute to the SSR [47]. A spatial response is seen with auditory stimuli tending to stimulate the auditory cortex, on the side contralateral to the stimulus [47], but the precise cluster of neurons constituting the location of the SSR source depends on frequency [44]. Although the early study of Galambos [43] seemed to imply the generation of a $40 \mathrm{~Hz}$ response regardless of stimulus, later studies indicate that the ERP is at the same frequency as the stimulus [46-49]. However, the $40 \mathrm{~Hz}$ frequency point does result in unique effects as would be expected from its association with processing of sensory information [36]. For example, a maximal superposition effect, with maximal SSR, is seen when using a $40 \mathrm{~Hz}$ stimulus as well as recruitment of activity from the cerebellum in certain cases $[47,49]$. The precise response generated is dependent on the nature of the auditory stimulus used.

Beats were used in [50] with disparate effects on EEG power and synchronization but usually a decrease in both parameters most pronounced when using monaural $5 \mathrm{~Hz}$ beat frequency and binaural $80 \mathrm{~Hz}$ beat frequency. Significantly, there was a maximal increase in EEG power when using a monaural $40 \mathrm{~Hz}$ beat frequency which may be linked to interneuron activity with these pathways possibly relevant in $\mathrm{AD}[27,50]$. This complex pattern of response when using beats as a stimulus source is also discussed in a 2013 study by Miyazaki et al. [64]. In [64], it was found that the SSRs measured using MEG showed both maxima and sometimes minima at the frequencies corresponding to the beat frequency. For example, maxima were reported for $3 \mathrm{~Hz}, 12 \mathrm{~Hz}$, and $40 \mathrm{~Hz}$ but minima reported at $8 \mathrm{~Hz}$ and $20 \mathrm{~Hz}$ (interestingly a $20 \mathrm{~Hz}$ beat frequency resulted in pronounced activity at the harmonic value of $40 \mathrm{~Hz}$ ).

An important final point from this review of auditory stimuli is that ERPs can be generated from endogenous sources which can then affect the response to an external stimulus. In [56], it was shown that SSRs resultant from FAM stimuli were more resistant to the effects of ERPs from endogenous sources than from click stimuli. This effect may have significance clinically as robustness and repeatability of response would be important in clinical use as the SSR generated should not be affected by variable endogenous factors if possible.

\section{Event related potentials from visual stimuli}

In this subsection, ERPs produced as a result of various visual stimuli are discussed, with findings that both complement those found in the studies which used auditory stimuli and offering further novel insights into the area of ERPs.

The 1995 study of Lutzenburger examined the concept of neuronal "coherent periodic activity" being responsible for cortical sensory processing with respect to visual stimuli [65]. In their work, the perifoveal area of participants was completely engaged by a monitor displaying bars moving in a random and then regular pattern. The regular pattern, of bars moving periodically downwards, was presented in either the upper, lower, left, or right halves of the visual field. EEG electrodes were placed over the part of the occipital lobe where the visual cortex was located as a square grid of $3 \times 3$ electrodes, encircled by 8 further electrodes with EEG activity during the stimulus period recorded. Interestingly, they calculated current source densities for their 17 electrode sites at the rear of the head instead of raw voltage because this allowed calculation of activity at the electrodes independent of the reference electrode. It also minimized distant source contributions to the signal while enhancing local brain contributions.

The electrical response recorded at each electrode was analyzed in three spectral bands with the mean normalized spectral power reported. With baseline power set as the response to the initial random pattern, only the $35-45 \mathrm{~Hz}$ band showed a significant change in response to the regular visual stimulus, with spectral power in this band increasing. In addition to this temporal response there was a spatial response, with the lower electrodes showing maxima response when the regular stimulus was in the upper part of the visual field and the upper electrodes showing the maxima when the regular stimulus was in the lower part of the field. This study demonstrated an increase in the power of gamma activity in electrodes placed over the visual cortex in response to an ordered visual stimulus with a consistent temporal and spatial response 
reported. There was a localized increase in gamma activity in that part of the visual cortex mapped to the area of visual field experiencing the coherent stimulus. In addition, the authors had previously reported a similar study with an increased response in gamma activity observed, albeit at $30 \mathrm{~Hz}$, to meaningful verbal stimuli as opposed to a much lower response to meaningless pseudowords [66].

The temporal and spatial response seen in this study is of note; there is an increase in spectral power at $35-45 \mathrm{~Hz}$ directly in response to the regular stimulus which maps to the part of the visual cortex linked to the part of the visual field stimulated. The study failed to demonstrate a spatial reaction for the left versus right regular stimulus, with an explanation being the shorter bar length used for that variant of the stimulus. The regular pattern in the study changed at a rate of $3 \%$, again demonstrating that a $40 \mathrm{~Hz}$ stimulus is not necessary to generate a $40 \mathrm{~Hz}$ neural response. However, it would have been of interest to see if a $40 \mathrm{~Hz}$ stimulus resulted in a larger response, as would be predicted from the superposition principle observed by Galambos et al. [43].

Responses to visual stimuli were studied by TallonBaudry et al. in a 1996 paper [67]. In this study, 8 participants had a 13 electrode EEG array located according to the 10-20 system, arranged symmetrically around the crown and posterior of the head, which recorded responses to stimuli. These stimuli included a real triangle, imaginary (Kanizsa) triangle, a "no triangle stimulus", and a distractor stimulus of a curved illusionary triangle. The latter was not included in the data analysis, but participants were asked to silently count the occurrence of this target. Eight blocks of 90 stimuli were delivered to each participant with each displayed on a video in random order for $700 \mathrm{~ms}$ at a visual angle of $2.5^{\circ}$ and at a distance of $2 \mathrm{~m}$. After rejection of epochs containing artefacts, each participant produced a mean of 154 responses per each of the three stimulus types. Analysis of the EEG signals produced revealed ERPs comprised of two distinct gamma frequency band components. One was produced about $90 \mathrm{~ms}$ after the stimulus, was phase locked to the stimulus, had a maximal evoked potential at the $\mathrm{Cz}$ and $\mathrm{C} 4$ electrodes, and did not vary with stimulation type. The second gamma frequency band component appeared later, at about $280 \mathrm{~ms}$ after the stimulus, and was not phase locked. More precisely, this second component comprised of two parts, one at $200-300 \mathrm{~ms}$ and another at $300-400 \mathrm{~ms}$. The overall second component had a diffuse location of maximal response, distributed approximately equally across the electrodes posterior to Pz. This diffuse location corresponds to the occipital lobe, location of the visual cortex. Further, the later component was greatest for the coherent triangle stimuli (real or imaginary) and weak (or negligible) for the no triangle stimulus.

From examining the results of [67], it appears that the gamma frequency band component occurring $90 \mathrm{~ms}$ after the visual stimulus correlates to that of the MLR observed by Galambos following auditory stimulation [43]. Pantev [44] also studied the MLR to auditory stimuli but in addition looked at a later response, which corresponds to the component occurring at about a $280 \mathrm{~ms}$ lag in the Tallon-Baudry study [67]. Pantev concluded it was this later component that was responsible for the maximal SSR [44]. The Lutzenburger study [65] focused on the response to visual stimuli and measured the overall evoked spectral power in the $35-45 \mathrm{~Hz}$ band, noting a temporal and spatial link to the stimuli but did not look at the individual components comprising this gamma band response as was done by Tallon-Baudry. In [67], it is speculated that the gamma band components to visual stimuli are linked to "feature binding" (the coherent perception of an object by the separate processing and then binding of the individual features) but notes the precise role of the earlier component is unclear. The later component was noted to be stronger for coherent triangles, which closely resembled to the target curved triangle. It is proposed in [67] that the stronger response may be due to a "matching mechanism" as the stimulus was processed and compared to the target. Hence this later gamma component may be linked interestingly to higher cognitive perception mechanisms. The diffuse locational nature of the later gamma component was ascribed to either deep and strong activity in a central processing location or rather multiple cortical locations.

In addition, in [67] it was found that these gamma band ERPs were found to have lower frequency $(0-25 \mathrm{~Hz})$ potentials occurring in complement to them. The lower frequency responses had slightly differing location characteristics, response characteristics to the stimulus type and time latency compared to the gamma components. Although these lower frequency potentials, along with the gamma band component, could be part of a broad band response to stimuli the differences in the nature of the two groups implies they are the products of neural activity from different locations, with presumably differing, but 
perhaps interlinked functions related to processing of visual stimuli.

Herrmann et al. examined the EEG response to visual stimuli of $1-100 \mathrm{~Hz}$ flicker at discrete $1 \mathrm{~Hz}$ steps [48]. Participants, 10 in total, were exposed to two white light-emitting diodes (LEDs), one in front of each eye in purpose-built goggles which illuminated the entire visual field. These LEDs flickered at each of the 100 frequencies in a pseudo-random order for $30 \mathrm{~s}$ at a time, with a $5 \mathrm{~s}$ pause in between. 19 tin EEG electrodes were placed according to the international 10-20 system with approximately 60 sequential $0.5 \mathrm{~s}$ epochs captured per discrete stimulus, which were then checked for artefacts. The spectral power was calculated at each frequency. The work investigated the idea that visual cortex neurons respond to a flickering stimulus at the same frequency as the flicker, which was confirmed with the finding that the so-called steady-state visual evoked potential response frequency had a strong fundamental at the same frequency as the stimulus with harmonic and sub-harmonic responses of the fundamental also present. This finding seems to go contrary to the idea expressed in the earlier paper of Galambos that a $40 \mathrm{~Hz}$ gamma response is evoked independent of stimulation frequency [43]; however, it does correlate with other studies, for example [47], and demonstrates the complexity of ERPs. The presence of harmonics and subharmonics does link though to the observation in [67] of lower frequency potentials occurring in complement to higher frequency components. The results of [48] also demonstrated a resonance phenomenon to some frequencies that may show neurons to have preferred frequencies. This resonance was seen at $10 \mathrm{~Hz}, 20 \mathrm{~Hz}, 40 \mathrm{~Hz}$, and $80 \mathrm{~Hz}$. The conclusion was that this resonance may explain the presence of predominant $40 \mathrm{~Hz}$ activity in perception of stimuli, as part of a complex non-linearly coupled system of many "neural oscillators". Further, [48] described the idea of "binding", how in order to coherently perceive an object, the features of the object, such as color or orientation, which are represented and processed in different parts of the visual cortex, are bound together. This binding process has been observed to happen predominantly at $40 \mathrm{~Hz}$ and involves both evoked (phase locked to the stimulus) and induced (not phase locked) gamma activity in response to the stimulus. The special nature of gamma frequency band in cognitive function and perception was also expressed in [48] with the observation that stimuli occurring at gamma frequencies are processed at a faster rate in human brains than those at other frequencies and are also bound better. A possible neuroanatomical explanation for the primacy of $40 \mathrm{~Hz}$ activity in perception was proposed with the basis in axonal connections between neurons. After every action potential, lag and feedback properties of the neurons in a group will result in a temporal synchronizing of activity between the neurons and a resultant preference for a $40 \mathrm{~Hz}$ oscillation. Finally, [48] demonstrated the visual stimuli used in this study resulted in a maximal response in the occipital lobe, location of the visual cortex, correlating with the spatial relationship between stimulus and response seen in the other earlier studies discussed earlier.

The three representative studies presented here, which are focused on ERPs produced from visual stimuli, add to the pool of knowledge on the fundamental physiology. Visual stimuli are seen to cause ERPs to be produced in the visual cortex [48, 65], and indeed discrete regions of the visual cortex can be activated preferentially depending on which part of the visual field is stimulated [65]. The ERPs produced may be complex in nature, comprised of evoked and induced responses varying in lag with respect to the stimulus and may be part of an overall broadband, diffuse response to processing of visual information $[48,67]$. Although SSRs seem to be produced at a frequency matching that of the stimulus [48], the gamma band seems to be particularly important. Power in the gamma band is increased significantly in response to visual stimuli [65]. Gamma activity may be linked to deep and strong central processing activity related to higher cognitive processing [67]. Further, neurons appear to have preferential operating frequencies, notably the $40 \mathrm{~Hz}$ point. Resonance is observed at $40 \mathrm{~Hz}$ and is related to complex "binding" processes involved in perception of visual stimuli, with faster and better binding thought to occur in response to stimuli presented at this frequency [48]. The spatial response, complex non-linear nature of the response and maximal effect seen in the gamma band and frequently at the $40 \mathrm{~Hz}$ point, complement the findings of the studies that involved auditory stimulation discussed in the previous section.

\section{Event related potentials from somatosensory stimuli}

The somatosensory system, a diffuse collection of receptor types and neural pathways that respond to a variety of stimuli including touch, pain, heat, vibration and pressure, is not as frequently studied as the more popular auditory and visual modalities. However, the somatosensory system may be an important 
channel for stimulation as it is usually left unaffected in neurological patients [68-70]. As such, it is worthy of discussion with external stimuli acting on this system being able to develop a typical steady state neural response, this time in the somatosensory cortex.

A 2014 study by Jamali et al. investigated vibration stimuli applied to the fingertips and the resultant response as measured by MEG [71]. An $8 \mathrm{~mm}$ inflatable plastic membrane was applied to a fingertip of participants with air pulses delivered at a frequency of $22.2 \mathrm{~Hz}$. These pulses were administered for $2 \mathrm{~s}$ (constituting a stimulus train), with a 1-1.5 s interval before repetition of the stimulus. In total, 90 stimulus trains were administered with a further 90 after an hour gap to 12 healthy participants. An SSR was produced in the somatosensory cortex on the side contralateral to the stimulus. Analysis of the SSR using bandpass filtering showed a response at the stimulation frequency and also in the gamma band at the harmonics of $44 \mathrm{~Hz}$ and also at $66 \mathrm{~Hz}$. It would be of interest to see if a response was produced at the subharmonic point of $10 \mathrm{~Hz}$; however, this frequency was removed by filtering. Of particular note was the observation in [71] that the $22.2 \mathrm{~Hz}$ response decreased over the course of a session, demonstrating habituation to the stimulus, whereas the gamma band responses was consistent over a session. Further, the pattern of the $22.2 \mathrm{~Hz}$ response was not different between sessions while the gamma band responses were larger in the later session. It is proposed in [71] that this pattern of gamma band response indicates neuroplastic change and an effect related to sensory binding and temporal organization of higher order processing; repeated stimulus experience resulting in an enhancement of temporal precision.

In another 2014 paper, this time by Pokorny et al. [68], the somatosensory system was targeted through the development of a device designed to produce tactile stimulation of mechanoreceptors present in skin. At the core of the system was a programmable microcontroller that delivered two independent stimulation signals via $\mathrm{C}-2$ tactors which are a standard actuator used in vibrotactile research [72]. The stimulation pattern consisted of a $200 \mathrm{~Hz}$ sinusoidal carrier modulated by either a rectangular signal of the stimulation frequency (called the sine tap stimulus) or amplitude modulated by a sinusoidal carrier (called the sine am stimulus). In a single sample experiment on a human participant, two C-2 tactors were attached to the two wrists of one healthy volunteer and tactile stimulation was delivered using the sine tap stimulus pattern at 7 discrete frequencies from $14-32 \mathrm{~Hz}$ in $3 \mathrm{~Hz}$ steps with 40 repetitions per frequency, to each wrist. The response was recorded using EEG electrodes covering the somatosensory cortex. It was found that an SSR was produced in the somatosensory cortex in the side contralateral to the wrist that was stimulated, with maximal responses produced in response to a $20 \mathrm{~Hz}$ stimulation frequency. Interestingly, $20 \mathrm{~Hz}$ is a subharmonic of $40 \mathrm{~Hz}$, which was not a frequency used in the study.

The somatosensory system may prove to be an important approach to creating ERPs in the brain for therapeutic use. Further, studies have shown the ability to establish SSRs using somatosensory stimuli such as touch and the ability to modulate these responses through methods such as user attention and interaction between multiple stimuli $[68,73,74]$. This implies the possibility of fine control and manipulation of a somatosensory induced SSR using a therapeutic device. Further control and permutations in the nature of the SSR may be possible through the type of somatosensory receptors activated and perhaps even the location on the body stimulated. For example, there are four distinct types of mechanoreceptors with different types of stimulation needed to activate each group. For instance, Merkel cells are responsive to static pressure and also low frequency tactile vibrations $(5-15 \mathrm{~Hz})$, Meissner corpuscles are responsive to vibrations in the $20-50 \mathrm{~Hz}$ band, with Pacinian corpuscles sensitive to higher frequency vibrations with maximal response in the $200-250 \mathrm{~Hz}$ band $[68,75]$. In [68], it is noted that a tactile stimulating device would need to cover the frequency range of $5-250 \mathrm{~Hz}$ to be capable of stimulating all the different types of mechanoreceptors. Finally, the work reported in [71] indicates a neuroplastic (brain remodeling) response to at least certain somatosensory stimuli and hence the possibility to induce a beneficial change in brain activity through exposure to the appropriate stimuli in an appropriate regimen.

\section{Brain stimulation using other external modalities of stimulation}

The most commonly used external modalities seen in studies relating to brain stimulation are those of auditory, visual, and to a lesser extent somatosensory. However, there is the possibility of using other approaches to generating activity in the brain, for example using external electrical current sources.

Transcranial electric stimulation refers to a group of non-invasive techniques used to stimulate the brain using electrical current. One subtype, that of transcranial alternating current stimulation (tACS), was the 
subject of a 2013 review article by Herrman et al. [76]. This review looked at this technology as a means of altering brain activity. It was noted at the start of the review that neural activities including cognitive functions are associated with brain oscillations of different frequencies (not necessarily in the gamma band), and that it may be possible to directly alter the oscillations and associated activity with external stimuli such as tACS. tACS in particular has the advantage of being frequency specific and hence should entrain brain oscillations only at the selected frequency, thus allowing close control. Interestingly the observation was also made in [76] that the links between neural oscillation patterns and function are usually correlative as opposed to causal but the use of emergent technologies such as tACS may help firmly establish causal links as well as facilitating beneficial manipulation of function. The physiological basis of the technology appears to be the direct modulation of neuronal firing to that of the applied electrical stimulus, entraining endogenous brain oscillations. The review contains listings of studies involving tACS applied to a range of neural activities that demonstrate, for example, the ability of the technology to enhance and inhibit motor cortex excitability, slow down and enhance voluntary movement and affect the visual cortex leading to a change in the detection of phosphenes by participants. Encouragingly, in some of the reported studies, there was evidence of the effect lingering for hours after removal of the stimulus. Such lasting effect would be vital for the use of any modality as a therapy.

Application of tACS at the $40 \mathrm{~Hz}$ frequency point were reported by [76] for some studies, for example [77-79]. In [77], the cortical excitability of the visual cortex was measured using tACS at a range of frequencies including $40 \mathrm{~Hz}$. This frequency did not significantly affect the visual cortex; however, the subharmonic value of $20 \mathrm{~Hz}$ did increase excitability. In [78], the effect of tACS on contrast sensitivity and contrast discrimination with respect to vision was assessed. The modality was found not to affect contrast sensitivity whereas discrimination was affected only by $60 \mathrm{~Hz}$ and not $40 \mathrm{~Hz}$ or $80 \mathrm{~Hz}$ tACS. Finally in [79], $40 \mathrm{~Hz}$ tACS was applied with $180^{\circ}$ phase difference between hemispheres and was found to affect the perception of bistable apparent motion stimuli; this effect was not seen with $0^{\circ}$ phase difference however.

An interesting area reported on by [76] was the use of combined DC and AC stimulation in studies concerned with memory and cognitive tasks [76, $80,81]$. It was found that stimulation of $<1 \mathrm{~Hz}$ applied during non-rapid eye movement (REM) sleep improved memory in participants. Next it was found that theta band stimulation of $5 \mathrm{~Hz}$ applied during non-REM sleep impaired memory with no effect on memory seen if the stimulus was applied in REM sleep or indeed during wakefulness. Thus, it was concluded in [76] that the effect of stimulation, on the cognitive domain in this case, depends also on the prevailing brain state (wakefulness, REM sleep, non-REM sleep) of the participant. This set of experiments clearly demonstrate the complexity involved in manipulating neural activity and causing an effective and controllable change with the state of the participant perhaps being a critical factor depending on the domain under investigation.

This complexity in modulating brain activity, using tACS again as an example, is further illustrated by the fact that robust protocols for implementation of tACS are not yet established with the experiments reported in the review by Herrmann varying widely in design and results [76]. Tailoring of amplitude, frequency, and phase may be necessary to elicit the desired change in neural activity, as well as other miscellaneous factors such as the participant's prevailing brain state as discussed, and as another example, the electrode setup. Regarding intensity, there may be a complex non-linear effect with inhibitory neurons more susceptible to stimulation than excitatory neurons as evidenced by studies reporting inhibition when using intensities of $0.2 \mathrm{~mA}$, excitation at $1 \mathrm{~mA}$ and no effect at intermediate values (when the two effects presumably cancel each other out). Further, stimulation intensity thresholds vary between individuals and may need to be factored in. Control of frequency is where tACS has an advantage over other technologies in that it is linked to one frequency. However, knowledge of the oscillatory frequency associated with the desired cognitive process is needed, with the stimulation frequency then set at that value. It is increasingly seen that phase is of importance, with for example the phase of theta oscillations able to modulate the amplitude of gamma oscillations. Hence derivation of an effective protocol, and indeed device, for the use of a sample modality such as tACS as a therapeutic tool to cause beneficial and repeatable effects should be theoretically possible.

\section{Stimulation from multiple sources}

In the studies presented so far, only one stimulation source at a time has been presented to a participant. Multiple sources of simultaneous stimulation result 
in an ERP and SSR influenced by all the sources used, which adds complexity but also presents opportunities to exert more control over the precise nature of the response generated. It is also noteworthy to reiterate that endogenous sources of stimulation, such as focusing on a task or reading, equally may trigger a response in addition to external sources. The effect of an auditory stimulus used concurrently with such endogenous sources was part of the study conducted by Voicikas et al. and is discussed above [56]. Presented below are other representative studies discussing the use of concurrent stimulation sources.

The SSR to a stimulus can be disrupted by the addition of a concurrent stimulus with a new, readjusted SSR established. This desynchronization of a SSR was the focus of a 2005 study by Ross et al., which looked at the $40 \mathrm{~Hz}$ response produced by auditory stimuli [82]. The study proposed that the SSR, which are gamma band oscillation patterns, and the response to a stimulus, are related to the processing and binding of a particular scene. A change in the stimulation pattern logically necessitates a change in the oscillation pattern to adjust to the new pattern of stimulation. This idea was studied using auditory stimuli comprised of a $40 \mathrm{~Hz} \mathrm{AM}$ of a $500 \mathrm{~Hz}$ tone, played to one ear, to evoke a $40 \mathrm{~Hz}$ auditory SSR and a concurrent short noise burst, in the $2-3 \mathrm{kHz}$ frequency range acting as the concurrent stimulus (or perturbation), to the contralateral ear and resultant response recorded using wholehead MEG. This dichotic stimulation experiment was then added to by a binaural variant where the initial stimulation pattern had the concurrent stimulus (perturbation) combined in. Finally, in a third experimental set, the periodicity of the stimulating AM signal was "violated" to act as the perturbation. It was found that the SSR, once established by the initial stimulus, was localized in the auditory cortices. The perturbation, regardless of type, resulted in a reduction of SSR amplitude followed by change in the amplitude and phase of the SSR which reflected a reset followed by establishment of a new SSR of synchronized gamma oscillatory activity. This interaction between initial stimulus and perturbation was not a simple superposition of individual responses, as was suggested by Galambos [43], as there was a reduction in SSR even when the perturbation was in phase with the initial stimulus. Rather, the SSR is a result of complex interaction with it being argued in [82] that the results indicate that the auditory SSR may be a stimulus driven oscillatory brain activity as opposed to an evoked response. Interestingly, it was proposed in [82] that the possibility existed of SSR interaction activity between stimuli of differing sensory modalities but hypothesized that strongest interactions would be between stimuli of the same type.

We feel this ability to adjust and tailor an SSR by selection of a set of concurrent stimuli may have therapeutic implications. Certain types of oscillation patterns may be more beneficial than others and tuning of these gamma band oscillatory responses produced in the brain appear to be achievable. The possibility of further fine tuning the oscillatory pattern by mixing stimulatory modalities would make such a therapy highly flexible and customizable.

DeLosAngeles studied SSRs generated as a result of states of meditation, as an endogenous source of stimulation, coupled with external stimuli in a 2010 study [83]. Three distinct external modalities were examined: auditory, visual, and somatosensory. The auditory stimulus was a $1500 \mathrm{~Hz}$ carrier wave amplitude modulated with a $40 \mathrm{~Hz}$ message frequency presented to both ears via pneumatic headphones. The visual stimulus was a light positioned $10 \mathrm{~cm}$ from the eye at a strobe frequency of $16 \mathrm{~Hz}$. Finally, the somatosensory stimulus was an electrical stimulus with a carrier frequency of $1500 \mathrm{~Hz}$ and a message frequency of $27 \mathrm{~Hz}$ applied to the wrist. These stimuli were presented separately in series to a set of meditators and non-meditators with a one-minute base period and one minute of exposure to stimulus conducted for each modality. This 6-minute protocol was repeated three times with an "attention condition" of "mind-wandering", "attend-to-breath", and "attend-to-stimulus" focused on in turn by each participant during each experimental set. EEG electrodes recorded the responses. Robust SSRs were produced in all participants. A spatial effect observed with each respective stimulus modality causing an SSR in electrodes positioned over the corresponding part of the cortex responsible for processing stimuli of that type. Further, the SSRs recorded showed a frequency response with the frequency of the stimulus mirrored in the EEG output along with evidence of harmonics. In [83], it was postulated that the amplitude of the SSRs would be affected by the attention condition of the participants, and that meditators would demonstrate a more pronounced effect than non-meditators. However, no modification of the SSR was seen with [83] concluding that modulation of SSRs require a high degree of attention, not realized in his experiment. This result suggests that adjustment of a SSR by the meditative state of a participant may not be 
possible, although it is noted [83] that a high degree of attention may be required to affect SSRs which may not have been achieved by the participants of the study.

This effect would not necessarily be a desirable in any case as it may lead to intra and inter- individual variation in response to a given stimulus as a result of a difficult to regulate confounding factor, that of the individuals' "state of mind". Rather, the creation and adjustment of an SSR purely by careful selection of external stimuli as alluded to in the Ross study [82] would, as a proposed therapeutic modality, be more repeatable and robustly applicable between patients.

A paper by Kosem et al. in 2014 examined presentation of visual and auditory stimuli concurrently to participants and the corresponding effect on neural oscillations, measured as event-related fields (ERFs) [84]. The motivation was to study time perception with respect to stimulatory input from the environment. Visual stimulation was presented to participants as discs lasting $16.7 \mathrm{~ms}$ while auditory stimulation was provided as white noise of $16 \mathrm{~ms}$ duration. These two stimuli were presented in blocks that had the two sources synchronized or with a $200 \mathrm{~ms}$ lag of one with respect to the other. A given block consisted of a stream of 65 stimuli, thus lasting about $1 \mathrm{~s}$, with multiple blocks presented over the course of the experiment differing in terms of the pattern of synchronization between blocks. The rate of presentation of blocks was $1 \mathrm{~Hz}$. Participants had their response recorded using MEG and also gave subjective feedback by reporting the order in which they perceived the stimuli. It was found that the visual and auditory stimuli caused ERFs in the visual and auditory cortices, respectively. Crucially, the ERF in the auditory cortex was found to be modulated, by means of a phase shift, as a result of the presence of the visual stimulus. This phase shift correlated to the participants' perception of simultaneity. In effect, the auditory cortex response was actively adjusted such that the timing of events in that cortex matched those of visual inputs. The participants initially perceived the stimuli as being out of phase, and then later reported perceiving them as being in phase (despite the stimuli still being out of phase). This perception correlated with the change of phase in the auditory cortex ERF caused by the visual cortex.

In the context of a theoretical treatment modality for $\mathrm{AD}$, it is of interest and perhaps therapeutic value to analyze the effect of presentation of multiple stimuli modalities concurrently. A complication may be that if the stimuli are not presented perfectly simultaneously; for example, if there was a variance in frequency, phase, or time lag, then there may be an impact on the resultant therapeutic neural oscillation patterns produced. An appreciation of the potential cross-talk in the oscillation patterns produced by the different modalities would also be needed. In [84], a visual stimulus was shown to modulate the ERP produced to an auditory stimulus by adjustment of phase, leading to changes in perception of timing. Hence, the study provides some evidence that the brain is able to compensate for, and handle, multiple stimulatory modalities delivered concurrently at least in order to adjust to timing of events from the environment. Specifically in [84], the brain was able to bring into alignment stimuli that are out of phase so to appear, subjectively, to be in phase. Also importantly, [84] showed that cross-talk, which may or may not a useful phenomenon, exists and would be a factor for consideration in a multiple modality therapy.

It is of interest to consider the effect of two stimuli presented together on the pattern of resultant ERPs and SSRs. The conclusion from the collection of studies presented here indicate that the effect is complex. The results of [83] imply that the SSR resultant from an external source of stimulation may possibly be resistant to interference from endogenous sources. However, in the Voicikas study [56], it was demonstrated that endogenous sources could in fact alter the SSR produced from a click based external auditory stimulus, with a FAM based stimulus more resistant to these endogenous sources. It would be desirable in a therapeutic modality that the SSR generated be solely influenced by the external stimuli used, as having to account for influence from difficult to control endogenous sources would lead to excessive complexity. It would also be of benefit if a tailored SSR could be generated using multiple simultaneous stimuli. The results of [82] indicate that simultaneous external stimuli do indeed lead to interaction in the brain and a composite SSR produced. Interestingly, [84] shows that this crosstalk may be intricate with, for example, visual stimuli causing a phase shift in the response generates to auditory stimuli with consequences on how individuals perceive timing and simultaneity. Indeed the results of [84] suggest that perhaps concurrent stimuli can be applied with the brain able to compensate for at least a partial difference in phase between the stimuli. This would render the practical application of simultaneous stimuli easier to implement. 


\section{Recording of electrical signals from the brain}

In most, if not all, of the above-mentioned studies, the neural activity of the brain is measured using EEG or MEG technology. Recordings from these technologies give insight as to the neurophysiology and detect with high time resolution changes in activity relating to function [42]. An important issue when using these technologies is the possibility of contamination of signal from sources other than the brain, with the main example in the case of EEG being electrical activity from muscle contraction in the form of electromyogram (EMG) signals. In order to stimulate the gamma activity in the brain, and to verify the stimulation effects, it will be vital to remove sources of contamination and noise artefacts impacting signals.

The 2016 study by Fitzgibbon et al. in particular investigated methods of removing such EMG artefacts from EEG signals [85]. EMG power can, for example, exceed EEG power by a factor of 10 in the $20-80 \mathrm{~Hz}$ band that roughly constitutes the gamma range $[85,86]$. Hence EMG signals constitute an important contamination source. In fact, in [85] it is remarked that (non-time-locked) gamma EEG cannot be measured reliably in humans, at least not without removal or compensation for such contamination.

An EEG scalp electrode in reality records a signal that is a linear mixture of neurogenic (ideally recorded by an EEG), myogenic (ideally recorded by an EMG) and other sources of interference such as electro-oculogram signals. The approach of independent component analysis (ICA) can separate the mixed signals from the two sources into independent components (ICs), hence allowing the discarding of the myogenic to leave theoretically the pure neurogenic component. The approach depends on an algorithm to separate a combined signal to produce purely EEG and EMG ICs followed by the identification and retention of the neurogenic part either manually or automatically [85]. EMG contamination can consist of the transient due to overt movement and the persistent due to continuous, mild, and variable muscle activity. It is the latter subtype of EMG contamination that was tackled in [85], with the development of an algorithm to automatically remove these components, although transient EMG artefacts were manually removed in a pre-processing step. The study used datasets from three separate groups and included the use of both visual (the visual stimulus using eyes closed at $16 \mathrm{~Hz}, 40 \mathrm{~Hz}$, and $50 \mathrm{~Hz}$ for 10 seconds) and auditory stimuli (the auditory dataset was taken from [83]) as well as some data from participants that had been paralyzed and hence free of EMG contamination on the resultant EEG.

The algorithm centered on the knowledge that EEG spectral power decreases with frequency [85, 87], while EMG spectral power in the (roughly) gamma range increases with frequency $[85,88]$. The gradient of the signal (slope of log of power to $\log$ of frequency) was analyzed with gradients decreasing at a faster rate than a threshold retained and assumed to be EEG, and others assumed to be EMG and rejected. The threshold was selected based on analysis of EEG spectra from participants that had been paralyzed pharmacologically and hence free of EMG contamination. Next, the algorithm and selected threshold was applied to EEG data from a variety of experimental groups that had used a range of stimuli including auditory and visual. It was found that the ICA approach, and in particular the algorithm developed in this study, robustly eliminated EMG contamination in the EEG signals up to about $50 \mathrm{~Hz}$. The automatic approach was found to be similar in efficacy to manual removal of the EMG artefacts and significantly this EMG removal resulted in a signal with the pure EEG cortical electrical activity preserved, enhanced, or even revealed [85]. In particular, and of interest in the context of $40 \mathrm{~Hz}$ stimuli, the removal of EMG contamination significantly cleaned up and revealed strong $40 \mathrm{~Hz}$ SSRs from visual and auditory stimulation of independent experimental groups [85]. These signals could confidently be interpreted as coming from brain sources. This study indicates that ICA is a powerful and effective tool to remove EMG contamination from EEG signals. Further, the study argues for the necessity of such filtering, especially if the band of interest is in the gamma range.

\section{Brain stimulation using external sources for clinical effect}

The previous section showed, with evidence from multiple studies, that the brain can be stimulated using a variety of external stimulatory modalities. Further, the neurophysiological response generated, the ERP and then SSR, although complex in nature is to an extent predictable and controllable. The motivation behind this review is the prospect that certain SSRs or patterns of SSRs may prove to be therapeutic in nature and could lead to benefit to people suffering from $\mathrm{AD}$ and other neurological conditions. If this is the case, then the challenge is to find which patterns of SSRs are therapeutic, what stimuli result in these 
patterns, and the optimum regimen of delivery for maximal therapeutic effect. The study of Iaccarino et al. [19] discussed in the third main section above gives considerable evidence to the idea that generation of gamma activity may lead to improvement in $\mathrm{AD}$. This proposed therapeutic effect of brain stimulation using external modalities of stimulation is discussed below in relation to $\mathrm{AD}$ in six cases (one focused on diagnostics) but also one other work related to dysphagia. Finally, at the end of this section, a discussion on deep brain stimulation (DBS) applied to AD is examined [89]. DBS, although clearly not a modality of external stimulation, is certainly related to the general paradigm of brain stimulation for clinical effect and has relevance in deepening the knowledge of the area.

An example of the ability to induce beneficial changes to neurophysiology for therapeutic effect (in dysphagic patients) by external electrical stimulus was described by Fraser et al. in 2002 [90]. This study demonstrated that deliberate and purposeful manipulation of the incredibly intricate anatomy and function of the brain is possible. The study concerned the concept of neuroplasticity, which is the ability of neurons to reorganize in terms of patterns of connections and activity with a consequent change in functionality. The application was that of pharyngeal motor activity, which is often compromised in stroke patients leading to dysphagia. The areas of the brain responsible for coordination of the complex act of swallowing are bilaterally arranged in the motor cortex and exhibit a unilateral functional dominance. If the dominant side is damaged then pharyngeal motor control is compromised and the undamaged, non-dominant side must remodel if recovery is to be achieved. In [90], an electrical stimulus was applied using a bipolar platinum ring pharyngeal electrode placed into a catheter and inserted trans-orally or nasally to reach the pharynx. Electrical stimulation was applied at a variety of frequencies (including $40 \mathrm{~Hz}$ ) for $10 \mathrm{~min}$ utes at a time, which preferentially activated sensory afferent neurons. The EMG response was assessed using transcranial magnetic stimulation, to assess the effect on the corticobulbar motor neural tracts. In 8 healthy participants, the researchers found a $5 \mathrm{~Hz}$ signal increased excitability in the tracts (interestingly $40 \mathrm{~Hz}$ was found to decrease activity), the intensity of the induced activity was directly related to the intensity of the applied stimulus and a 10-minute period of stimulation resulted in an increase in excitability in the tract that reached a maximal value $60-90$ minutes after the end of the stimulation. Detailed mapping of the corticobulbar tract in a number of the participants using transcranial magnetic stimulation before and after pharyngeal stimulation showed an increase in the pharyngeal response area as measured on the scalp, with the increase observed bilaterally but of greatest size in the dominant hemisphere. Further investigation using functional MRI showed an increase in blood supply to areas of the brain associated with swallowing one hour after the end of stimulation. This functional MRI result implied that the electrical stimulation was causing a functional change in the part of the motor cortex responsible for pharyngeal activity.

Next, and of most interest, was the results of applying the pharyngeal electrode stimulus to 10 dysphagic stroke patients (an additional 6 patients received sham treatment). In the healthy cohort, the stimulation had no effect on actual swallowing performance. However, in the dysphagic group receiving treatment swallowing activity was improved as measured by a reduction in pharyngeal transit time, swallowing response time and of clinical significance, an improvement in aspiration score. These measurements were performed one hour after stimulation with comparison to, and improvement on, measurements taken before stimulation. Hence, [90] demonstrated an ability to affect the excitability of motor nerves serving the pharynx through stimulation patterns applied to sensory neurons. Further, [90] demonstrated objective clinical benefit to dysphagic patients that lasted at least one hour (with a suggested duration of 2-3 hours) after the end of the stimulation period. In [90], it was believed that the effect was mainly due to induced change in the cerebral cortex and was as a result of cortex plasticity. One drawback, however, was that the change in neural activity for the dysphagic cohort occurred in the undamaged hemisphere, with little if any change seen in the damaged side. Nonetheless, this study demonstrates that external stimulation applied to sensory pathways can alter brain activity, through the medium of neuroplasticity in this case, with resultant therapeutic effect possible.

Da Silva et al. conducted a review in 2015 into the use of light and sound stimulation as a therapeutic modality [91]. It is perhaps suggestive of how new this field is that only four studies were found and reported on; indeed, it was noted in [91] that light and sound stimulation are the least tested modalities of brain stimulation. Two of the studies were concerned with sports performance, with the other two health care related with one being a case report. Of interest, in the review was one study conducted by 
Vieira et al. which investigated the use of light and sound stimuli in 37 patients of AD [92]. Visual stimulation was provided through strobing LED lamps fitted onto the inner lens of sunglasses, while auditory stimulation was binaural beats through headphones. The two sources were combined to deliver the stimulation at specific frequencies, reported to be in the range $1-30 \mathrm{~Hz}$. The stimulation was associated with activities dependent on high levels of memory and it was found that an increase in both performance and cortical performance (as measured by EEG) was evident as a result of the treatment, as compared to a control group. It was postulated in [92] that the therapeutic effect achieved was possibly the result of neuroplastic processes, the ability of the brain to remodel in response to stimulation. A similar neuroplastic hypothesis is proposed in [90]. It was mentioned in [92] that photic and auditory stimulation may be applicable as a method to prevent and treat conditions such as AD.

It is thought that $40 \mathrm{~Hz}$ activity in the brain is related to cognition and is associated with healthy brain activity [93-95]. Further, patients with AD may have lower levels of $40 \mathrm{~Hz}$ activity compared to normal [93, 94]. As such, $40 \mathrm{~Hz}$ appears to be a natural choice of frequency with which to apply stimuli to AD patients. The 2016 study of Clements-Cortes et al. investigated the use of somatosensory stimuli, at $40 \mathrm{~Hz}$, directly applied to patients of $\mathrm{AD}$ [93]. The stimulus was applied using rhythmic sensory stimulation (RSS) which acts by deeply stimulating mechanoreceptors. Specifically, the device used was the NextWave chair [96], which produced sinusoidal $40 \mathrm{~Hz}$ sound waves through 6 speakers that resulted in vibrotactile stimulation of the whole body of patients. The study consisted of 18 patients all with diagnosed $\mathrm{AD}$ (6 mild, 6 moderate, and 6 severe). These patients underwent 6 sessions of RSS therapy, each session lasting 30 minutes. The amplitude, direction of movement, and pressure of the sound waves varied both throughout the session and at the different speaker locations. The frequency was nominally $40 \mathrm{~Hz}$ but varied from $39.96 \mathrm{~Hz}$ to $40.06 \mathrm{~Hz}$ in order to avoid the mechanoreceptors becoming habituated and unresponsive. The patients also had 6 sessions of visual stimulus treatment, while sitting with the chair turned off, of DVDs of relaxing scenes produced for AD patients [93]. Sessions were given twice weekly for six weeks with a wash-out time of at least 2 days before crossover from one type of treatment to the next. The efficacy of both modalities of treatment was assessed using standardized scoring systems such as the Saint Louis University Mental Status (SLUMS) [97]. The study found that there was a statistically significant difference in the effect of the two modalities with the RSS treatment giving on average an improvement of 0.5 units in SLUMS score per session, while the visual treatment had no effect on the SLUMS score of the patients. It was suggested in [93] that these results implied that $40 \mathrm{~Hz}$ stimulation could lead to increased cognition, with largest impact found in the mild to moderate $\mathrm{AD}$ cohort. This study uniquely provided a behavioral endpoint, that of the SLUMS scoring system.

Neural gamma activity at $40 \mathrm{~Hz}$ was not measured in patients in [93]. Although this was not the focus of the study, it would be interesting to determine if such a measure, by EEG or other means, would give an indication as to whether improved cognition was associated with a change in $40 \mathrm{~Hz}$ activity and if so where in the brain this change was happening. Furthermore, it would have been of interest to assess if prolonged, or more frequent, treatment would have resulted in an increase of sufficient magnitude in the SLUMS score of patients to re-classify them as normal. However, the results of the study were encouraging and provide solid evidence for the efficacy of $40 \mathrm{~Hz}$ stimulation treatment in $\mathrm{AD}$ patients. In addition, the fact that the somatosensory system is usually undamaged in neurological patients [68-70] makes the outcomes of [93] of particular interest. The results reported in [93], and also in the related publications of $[98,99]$, indicate that RSS has potential as an effective modality for exploiting the somatosensory system for therapeutic effect in $\mathrm{AD}$.

There is a further noteworthy technique, closely related to RSS, called vibroacoustic therapy (VAT). VAT is a multimodal approach involving sinusoidal low-frequency sound waves pulsed through a bed or chair specially designed for this purpose [100]. In VAT, the frequency of the sound $(20-100 \mathrm{~Hz})$, length of the pulsation, volume, and scanning of the sound (change in the precise frequency of the sinusoid) can be adjusted to achieve optimal effect as judged by the therapist. The modality may also feature music as an added component. The physiological basis behind VAT may relate to a resonance effect in the body, activation of particular receptors including like the Pacinian corpuscle and a "cleaning effect" of vibration on the body [100]. It has been applied to patients with chronic pain conditions, as well as patients with Parkinson's disease [100]. In [101], the idea that conditions like $\mathrm{AD}$ may feature a disturbance in 
oscillatory activity that may be responsible for some of the pathology is postulated. Electrical stimulation of the brain, regulating the oscillatory activity may aid in treating such conditions and such appropriate stimulatory patterns may be delivered using VAT for therapeutic effect [101].

The use of tACS as a diagnostic tool in AD was explored in a 2016 study by Naro et al. [102]. The diagnosis of AD and the related, and sometimes precursor, condition of MCI rely heavily on neuropsychological tests for both diagnosis and identification of those MCI patients likely to develop AD $[102,103]$. The pattern of brain oscillations, particularly in the gamma band (referred to as gamma-band oscillations (GBOs) in the study) and in certain locations like the cortical-thalamocortical network were postulated to be useful as a focus for a diagnostic tool based on tACS. The study consisted of 35 $\mathrm{AD}, 25 \mathrm{MCI}$, and 27 healthy control participants. Each participant underwent a range of neurophysiological tests and EEG recording at the start of the study. EEG recording to assess GBO power was performed at various sites in accordance with the 10-20 international system. Next, a tACS conditioning protocol was administered separately (on different days) over certain areas of the brain all on the left hemisphere, including the dorsomedial prefrontal cortex. The tACS setup consisted of a rectangular active electrode at the site of interest on the left hemisphere and a reference electrode over the right mastoid. Stimulation comprised of a 10-minute period with gamma frequencies from 40-120 Hz. After tACS stimulation, another range of neurophysiological tests was performed along with EEG performed at that time and one hour later. The two tests of neurophysiological tests and EEG were again performed at follow-up 2 years later.

It was found that $\mathrm{AD}$ patients generally performed worse in the neuropsychological tests than the MCI patients. Next, the GBO power immediately and one hour after tACS was found to increase in the control participants as compared to before tACS stimulation. GBO power partially increased in 21/25 MCI patients and did not increase in any of the AD patients. Further, at 2-year follow up, the control participants and MCI patients who exhibited an increase in GBO remained stable while the 4 MCI patients who did not show an increase in GBO power originally had by now developed AD. Hence this study demonstrated that tACS could be used to differentiate between AD and MCI and also identify MCI patients at high risk of developing AD.
Application of light and sound stimuli on clinical patients was reported in a study by Calomeni et al. [104]. Different cohorts were involved in the study, with two groups (consisting of 15 participants each) being elderly participants without dementia and patients with diagnosed $\mathrm{AD}$. The groups were exposed to light and sound stimulation in the low frequency alpha band at discrete frequency points including $8 \mathrm{~Hz}, 10 \mathrm{~Hz}, 12 \mathrm{~Hz}, 14 \mathrm{~Hz}$, and $15 \mathrm{~Hz}$, with EEG measurements recorded. Cognitive testing was done before and after stimulation. Altogether, 10 stimulation sessions, each of 15 minutes total duration, were conducted over a 20-day period on the groups. The study showed an increase in average alpha wave activity after stimulation in both cohorts, with some evidence of improvement in cognitive performance in all participants.

It is not clear whether the stimuli were administered separately or concurrently. Further only one type of cognitive test, the digit span test, was used. This test involved recalling a sequence of numbers. A confounding factor in this test is the reporting in [104] of a "training" of 15 minutes after stimulation in relation to this test, which may have impacted the results. Despite this, the study of [104] concludes that the results of the study supports the possibility of a therapeutic modality acting by modulation of brain activity and neuroplasticity.

The gamma band response (taken as $25-48 \mathrm{~Hz}$ ) of $39 \mathrm{AD}$ patients and 21 normal participants to two different visual stimuli was studied by Basar el al. [105]. The AD patients were further subdivided into those receiving pharmacological treatment and those not. The study aim was to investigate the SSRs (measured as EEG coherences) to a sensory visual stimulus and event related visual stimulus. An EEG coherence effectively is a measure of the temporal synchronization of the EEG signals as recorded at electrode pairs [106]. In [105], the sensory stimulus was of a white screen that flashed on and off intermittently. The event related stimulus was an odd-ball paradigm where a target appeared on a screen randomly and at intervals with all participants instructed to count the target appearances. Both of these stimuli were presented for $1 \mathrm{~s}$ with intervals of between 3-7 s between presentations. The SSRs were recorded using EEG scalp electrodes with the responses calculated for a range of intrahemispheric electrode pairs. The results of the study found that both the sensory visual stimulus and event related visual stimulus caused an increase in gamma band activity, including specifically at $40-48 \mathrm{~Hz}$, in all individuals with a significantly larger 
response in the $\mathrm{AD}$ patients compared to the normal participants. Further, the increase in gamma response was not affected by the presence or absence of concurrent drug therapy in the AD cohort. Interestingly, this increase in gamma activity was caused by stimuli not at $40 \mathrm{~Hz}$ and also the event related stimulus could be interpreted as a cognitive stimulus, which would be another potential type of stimulus modality.

In [105], it is noted that gamma activity is related to a wide variety of cognitive function and has a role in a variety of related processes such as memory, emotion, attention, and perception. It is also noted that AD patients have abnormal gamma activity, with an element of controversy in the literature as to whether gamma levels are decreased $[36,37,105]$ or indeed increased [107] in the condition. In [105], hypotheses to explain this finding of abnormal gamma activity are proposed with credence given to an impairment to inhibitory neurons. Inhibitory neurons produce gamma activity and abnormalities in this functionality may be relevant to the pathogenesis [36]. The involvement of inhibitory neurons may be also related to GABAergic activity and cholinergic balance which would relate it to other AD models [19, 27, 105]. The results of [105] provide for the application of the observed changes in gamma activity and in particular the topological patterns of change as recorded on the EEG, to a potential diagnostic tool, potentially neuroimaging, for AD. However, the findings are in keeping with the overall thrust of this review in that gamma activity appears to be decreased in AD patients (this controversial premise is discussed below in the section on limitations), and it is possible to increase these levels, hopefully to normal levels, through external stimuli. Again, as with other studies, a cognitive end point would have been of value, but it was not the aim in this case.

The Tsai laboratory at the Massachusetts Institute of Technology, which was responsible for the Iaccarino study [19], has produced some exciting new developments in the application of $40 \mathrm{~Hz}$ stimulation to AD patients [108, 109]. Some of these advances were presented at a recent conference of the Society for Neuroscience [110], which indicated that external sound stimuli at $40 \mathrm{~Hz}$ may cause a reduction in $\mathrm{A} \beta$ levels in mice in both the auditory cortex but crucially also with evidence of this effect in the hippocampus. The proximity of the auditory cortex and hippocampus may be a factor in this effect. The ability to target and affect change in the hippocampus by external stimuli would be an exciting development as the hippocampus is a common site of neuron loss in $\mathrm{AD}$ [10].
A spin-out company, "Cognito Therapeutics", which involves members of the Tsai laboratory, has begun trialing gamma wave therapy in 12 mild and moderate AD patients with a mixture of visual, auditory, and somatosensory modalities [111].

The clinically applied use of brain stimulation is quite a young field with few studies as yet. However, the promise of efficacy is there and motivates further work in the area. Definite therapeutic effect is possible, as reported in [90]. With regards to $\mathrm{AD}$, although the pathology of $\mathrm{AD}$ is intricate, complex, and not yet fully understood, new therapeutic options for the condition are an active area of research. Gamma activity is important for cognitive and related functions such as memory and may be reduced in AD patients [18, $36,37,105]$. Reports such as $[19,108]$ seem to indicate that gamma-based therapy is a non-invasive and relatively safe modality with promising efficacy in mouse models. This promise is added to by studies such as [93] which relate gamma-based therapy to cognitive end points. Table 1 below summarizes the papers in this section that involved $\mathrm{AD}$ patients in the course of the respective study. The table lists information on the nature of the stimuli used and the resultant effect seen. The details in this table may aid selection of stimuli and protocols in future work in the area.

Further insights into the harnessing of brain stimulation to elicit therapeutic effect is seen in the review of Mirzadeh et al. relating to the use of DBS in AD [89]. AD is modelled by the authors as a neural circuit disorder (in addition to the typical neurodegenerative model) with circuits serving memory and cognition seen as having high levels of dysfunctionality. A target for DBS was the nucleus basalis of Meynert (NBM) which features atrophy in AD with consequent effects on cholinergic innervation of areas such as the hippocampus [89]. DBS of the NBM was found to improve cognitive function in a single patient with Parkinson's disease dementia $[89,112]$ but did not have a significant effect when trialed on AD patients (though some evidence of a slowing of disease progression was reported) $[89,113]$. However, what was found, and had been noted before [84, 109] was that glucose metabolism was elevated as a result of DBS as measured by FDG-PET indicating an increase in the metabolism, and hence activity, of the neural tissue as a result of the treatment $[89,113]$. DBS applied to the hypothalamus was found to enhance memories in a non-AD obese patient, with the effect repeatable $[89,114]$. Further study into DBS of specifically the fornix of the hypothalamus cautiously indicated a promising well-tolerated therapy giving a reduction 


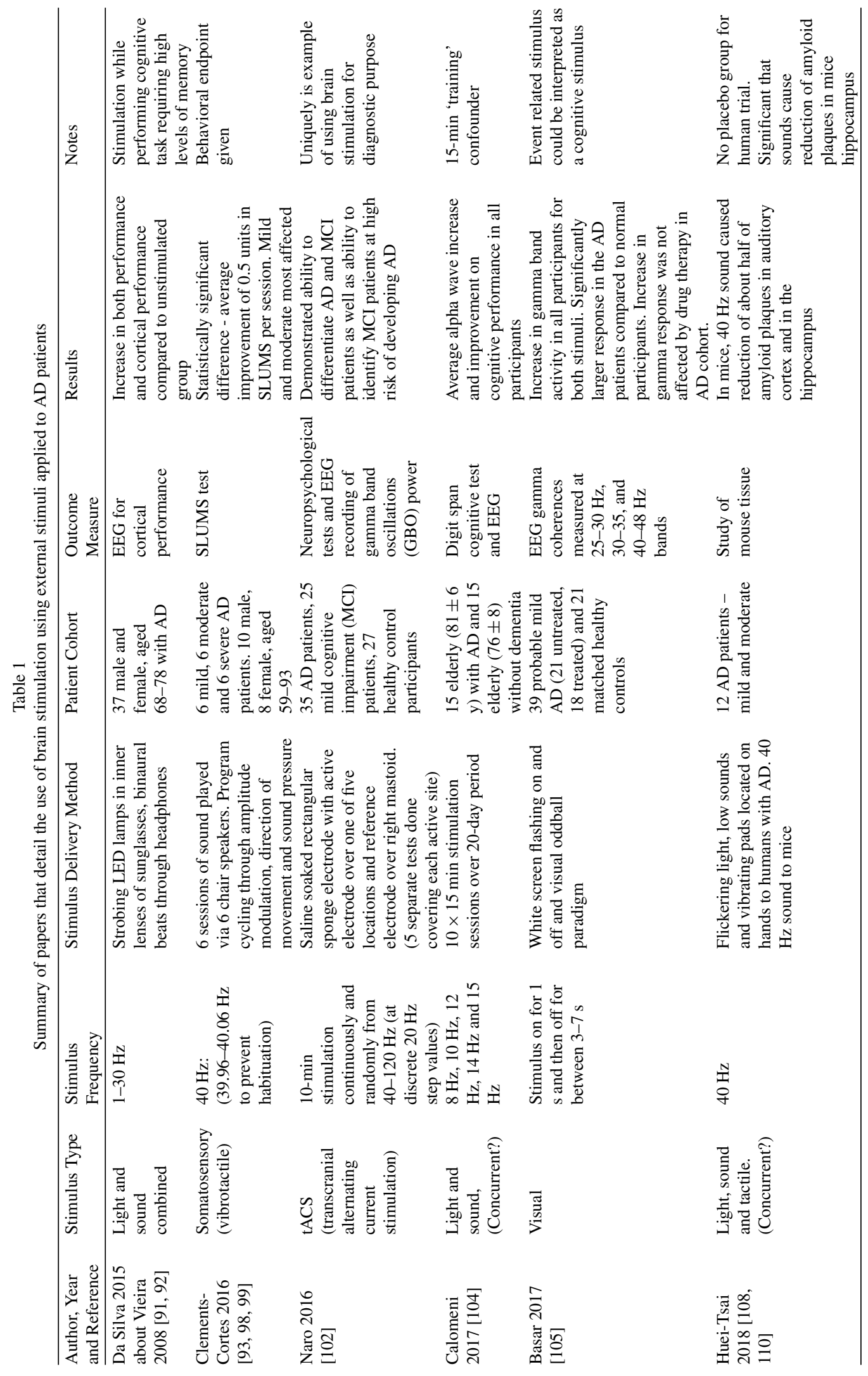


in the rate of decline (if not improvement) of $\mathrm{AD}$ [89, 115]. Further, DBS of the fornix results in positive changes to the neural physiology and anatomy, with activation of neural circuits linked to memory and an increase in hippocampal volume $[89,115,116]$.

These results from DBS indicate that appropriate neural stimulation, targeted to the correct location can result in physiological, anatomical and crucially, clinical improvement. Although successful application of an external stimulation modality (or modalities) may be more challenging to implement, the advantage of non-invasiveness makes it a worthy target to achieve.

\section{Limitations of brain stimulation using external sources}

There are potential limitations to the external gamma-based brain stimulation as a therapy for AD, as well as in the studies performed in this area so far. The limitations that have been identified to date are listed below.

- The underlying mechanism of action of gammabased stimulus on the molecular and cell level is not yet fully elucidated and gaps in this understanding may hide impediments to clinical efficacy. The study described in [19] showed a reduction in $A \beta$ as a result of a neurogenic and microglial mediated response and also a reduction in p-tau with indications of a microglial response. As described earlier, $A \beta$ and tau, although pathognomonic to $\mathrm{AD}$ do not fully describe the pathology and removal of these proteinaceous materials may not necessarily result in improvement of symptoms or cure.

- Microglial activation, which is desirable in context of the results of [19], may also be toxic if excessive. This issue is reported in [117], microglia may aid in the clearance of $A \beta$ as well as releasing neuroprotective growth factors but equally if over stimulated may trigger a disproportionate reaction and cause inflammatory damage. This over-reaction may also be more likely in older patients where microglia are less efficient [117].

- The treatment may be contraindicated in patients with epilepsy due to the possible epileptogenic nature of some of the stimuli $[48,118]$.

- Compliance with an external stimulus as a treatment regimen may also be an issue. For example, in [19], the treatment sessions of 1 hour visual stimulation gave an effect lasting 12-24 hours, while in [93] twice weekly 30 minute sessions were used with a somatosensory stimulus. These treatment lengths are unarguably more inconvenient than taking daily pharmaceutical oral dosage forms. Further, the nature of the treatment may not be acceptable for some patients as it may be subjectively irritant. However, these issues may be ameliorated by the finding in [49] that a response may be observed even if the patient is asleep (although perhaps with a lower level of response).

- Targeting of a particular part of the brain using an external stimulation modality may be challenging to achieve. For example, DBS studies have shown the fornix of the hypothalamus to be a more promising target than say the NBM [89]. Deliberate focusing of stimulation to such sites starting from an external source will present obstacles not applicable to more invasive modalities like DBS.

- Most important, there is a lack of sufficient studies using the modality in humans with firm cognitive end points demonstrating efficacy. The studies that have been conducted in AD patients are summarized in Table 1.

- Finally, there is controversy as to whether AD patients have a specific deficit in gamma activity. This point was briefly alluded to above while discussing the 2017 work of Basar [105], and is addressed in more detail here. The 1991 study of Ribary et al. demonstrated a reduction in the $40 \mathrm{~Hz}$ electrical activity particularly at the cortical level of AD patients exposed an auditory stimulus as measured using magnetic field tomography [94]. A 2002 study by Stam et al. demonstrated a loss in gamma band synchronization in $\mathrm{AD}$ patients (as well in other bands) using MEG [119] with these findings supported by a 2005 EEG based study by Koenig et al. [120]. However a 2006 study again by Stam et al. adds to the complexity of the picture by reporting, for $\mathrm{AD}$ patients, no significant correlation between gamma band synchronization and $\mathrm{AD}$ severity (as measured using the Mini-Mental State Examination) as well an increase in coherence in the gamma band for $\mathrm{AD}$ patients when again using MEG [121]. Some more recent studies have supported this finding of an increase in the gamma band power in $\mathrm{AD}$ patients $[107,122$, 123]. It is hence unclear if $A D$ features a gamma band deficit or excess compared to normal. It is likely that the neurophysiology is complicated 
and features disturbances in the overall network with deficits in normal neurological pathways $[36,122]$.

Although a presumed deficit in gamma band activity is central to the hypotheses of Iaccarino et al. (with indeed reduced gamma power in the 5XFAD mouse model used by the researchers) [19], it is possible that external gamma stimulation if supplied according to a correct regimen may cause therapeutic effect by modulating back to normal the abnormal pattern of activity seen in AD patients be that increased or decreased. However, there is a need for research into the precise nature of gamma activity disturbances in AD patients to facilitate development of optimal therapies based on the idea of external stimulation.

Ultimately some of these limitations may be overcome or deemed acceptable if an optimum treatment regimen is designed and sufficient clinical efficacy is demonstrated.

\section{CONCLUSIONS}

$\mathrm{AD}$ is a debilitating condition with a complex, as yet only partially understood pathology, and is lacking in efficacious treatment options. This review has examined the use of brain stimulation as a potential novel therapeutic modality for the condition. External stimulatory modalities such as auditory, visual, and somatosensory cause the responsive generation of brain electrical activity as ERPs and consequent SSRs. These patterns of brain activity although complex in nature, are to an extent predictable, reproducible, and may theoretically be tailored and targeted to certain areas of the brain, at certain frequencies and power levels through the use of appropriate stimuli or mixtures of stimuli. Therapeutic effect for a variety of conditions featuring a neurological component may be possible through the generation of such electrical activity.

Of particular interest are stimulation sources in the gamma frequency band, and in particular the $40 \mathrm{~Hz}$ point. Gamma electrical activity is associated with cognitive and other related activities with a disturbance in normal patterns of activity seen in patients of $\mathrm{AD}[18,36,107]$. The $40 \mathrm{~Hz}$ frequency value seems of particular neurological importance and as such represents a natural target value $[19,43,47,48,50,56]$. Stimulatory sources acting in this band, and resultant ERPs and SSRs do indeed show promise for clinical application to $\mathrm{AD}$ with studies reporting efficacy in both mouse models of the disease [19] and cognitive end points when applied to humans [93].

Despite possible limitations, therapy based on external stimulation represents an exciting nonpharmacological and minimally invasive approach to the tackling of AD. As such, gamma-based therapies are a worthy area for further research and development and may in the future form part of a core therapy for the disease or be part of a multimodal approach. This review has presented, through relevant papers, a discussion of the underlying neurophysiology, along with early studies on clinical applications, and seeks to accelerate the development of this promising area. Areas of future research should include refinement of the stimulus parameters, optimization of the stimulation modality or indeed possibly simultaneous modalities, and more studies with cognitive end points to demonstrate clinical efficacy. Ultimately, the objective of such research would be the development of a therapeutic device that shows effective prevention and treatment of $\mathrm{AD}$ through robust objective and purposeful modulation of gamma neuronal activity in people.

\section{ACKNOWLEDGMENTS}

The research leading to these results has received funding from the European Research Council under the European Union's Horizon 2020 Programme/ ERC Grant Agreement BioElecPro n.637780, Science Foundation Ireland (SFI) grant number 15/ER CS/3276, the Hardiman Research Scholarship from NUIG, the charity RESPECT and the People Programme (Marie Curie Actions) of the European Union's Seventh Framework Programme (FP7/20072013) under REA Grant Agreement no. PCOFUNDGA-2013-608728.

Authors' disclosures available online (https:// www.j-alz.com/manuscript-disclosures/18-0391r2).

\section{REFERENCES}

[1] Reitz C, Brayne C, Mayeux R (2011) Epidemiology of Alzheimer disease. Nat Rev Neurol 7, 137-152.

[2] Scheltens P, Blennow K, Breteler MMB, de Strooper B, Frisoni GB, Salloway S, Van der Flier WM (2016) Alzheimer's disease. Lancet 388, 505-517.

[3] Kumar A, Singh A, Ekavali (2015) A review on Alzheimer's disease pathophysiology and its management: An update. Pharmacol Rep 67, 195-203.

[4] National Institute for Health and Care Excellence. Dementia assessment and diagnosis. https://pathways.nice.org. uk/pathways/dementia/dementia-assessment-anddiagnosis. Accessed on February 28, 2018. 
[5] McKhann G, Drachman D, Folstein M, Katzman R, Price D, Stadlan EM (1984) Clinical diagnosis of Alzheimer's disease: Report of the NINCDS-ADRDA Work Group under the auspices of Department of Health and Human Services Task Force on Alzheimer's Disease. Neurology 34, 939-944.

[6] McKhann GM, Knopman DS, Chertkow H, Hyman BT, Jack CR, Kawas CH, Klunk WE, Koroshetz WJ, Manly JJ, Mayeux R, Mohs RC, Morris JC, Rossor MN, Scheltens $\mathrm{P}$, Carrillo MC, Thies B, Weintraub S, Phelps $\mathrm{CH}$ (2011) The diagnosis of dementia due to Alzheimer's disease: Recommendations from the National Institute on Aging-Alzheimer's Association workgroups on diagnostic guidelines for Alzheimer's disease. Alzheimers Dement 7, 263-269.

[7] Johnson KA, Fox NC, Sperling RA, Klunk WE (2012) Brain imaging in Alzheimer disease. Cold Spring Harb Perspect Med 2, a006213-a006213.

[8] Iverson DJ, Gronseth GS, Reger MA, Classen S, Dubinsky RM, Rizzo M (2010) Practice Parameter update: Evaluation and management of driving risk in dementia: Report of the Quality Standards Subcommittee of the American Academy of Neurology. Neurology 74, 1316-1324.

[9] Rayment D, Biju M, Zheng R, Kuruvilla T (2016) Neuroimaging in dementia: An update for the general clinician: Neuroimaging in dementia. Prog Neurol Psychiatry 20, 16-20.

[10] Kepp KP (2016) Alzheimer's disease due to loss of function: A new synthesis of the available data. Prog Neurobiol 143, 36-60.

[11] Coppola G, Di Renzo A, Ziccardi L, Martelli F, Fadda A, Manni G, Barboni P, Pierelli F, Sadun AA, Parisi V (2015) Optical coherence tomography in Alzheimer's disease: A meta-analysis. PLoS One 10, e0134750.

[12] Shah TM, Gupta SM, Chatterjee P, Campbell M, Martins RN (2017) Beta-amyloid sequelae in the eye: A critical review on its diagnostic significance and clinical relevance in Alzheimer's disease. Mol Psychiatry 22, 353-363.

[13] Ovod V, Ramsey KN, Mawuenyega KG, Bollinger JG, Hicks T, Schneider T, Sullivan M, Paumier K, Holtzman DM, Morris JC, Benzinger T, Fagan AM, Patterson BW, Bateman RJ (2017) Amyloid $\beta$ concentrations and stable isotope labeling kinetics of human plasma specific to central nervous system amyloidosis. Alzheimers Dement 13, 841-849.

[14] Nakamura A, Kaneko N, Villemagne VL, Kato T, Doecke J, Doré V, Fowler C, Li Q-X, Martins R, Rowe C, Tomita T, Matsuzaki K, Ishii K, Ishii K, Arahata Y, Iwamoto S, Ito K, Tanaka K, Masters CL, Yanagisawa K (2018) High performance plasma amyloid- $\beta$ biomarkers for Alzheimer's disease. Nature 554, 249-254.

[15] National Institute for Health and Care Excellence. Dementia overview. https://pathways.nice.org.uk/path ways/dementia/dementia-overview, Accessed on February $28,2018$.

[16] de Bruijn RF, Bos MJ, Portegies ML, Hofman A, Franco $\mathrm{OH}$, Koudstaal PJ, Ikram MA (2015) The potential for prevention of dementia across two decades: The prospective, population-based Rotterdam Study. BMC Med 13, 132.

[17] Kurz A, Perneczky R (2011) Novel insights for the treatment of Alzheimer's disease. Prog Neuropsychopharmacol Biol Psychiatry 35, 373-379.

[18] Fitzgibbon S, Pope K, Mackenzie L, Clark C, Willoughby $\mathrm{J}$ (2004) Cognitive tasks augment gamma EEG power. Clin Neurophysiol 115, 1802-1809.
[19] Iaccarino HF, Singer AC, Martorell AJ, Rudenko A, Gao F, Gillingham TZ, Mathys H, Seo J, Kritskiy O, Abdurrob F, Adaikkan C, Canter RG, Rueda R, Brown EN, Boyden ES, Tsai L-H (2016) Gamma frequency entrainment attenuates amyloid load and modifies microglia. Nature 540, 230235.

[20] Hardy J (2002) The amyloid hypothesis of Alzheimer's disease: Progress and problems on the road to therapeutics. Science 297, 353-356.

[21] Hardy J (2009) The amyloid hypothesis for Alzheimer's disease: A critical reappraisal: Amyloid hypothesis for Alzheimer's disease. J Neurochem 110, 1129-1134.

[22] Huang Y (2011) Roles of apolipoprotein E4 (ApoE4) in the pathogenesis of Alzheimer's disease: Lessons from ApoE mouse models. Biochem Soc Trans 39, 924-932.

[23] Maccioni RB, Farías G, Morales I, Navarrete L (2010) The revitalized tau hypothesis on Alzheimer's disease. Arch Med Res 41, 226-231.

[24] Kametani F, Hasegawa M (2018) Reconsideration of amyloid hypothesis and tau hypothesis in Alzheimer's disease. Front Neurosci 12, 25.

[25] Lambracht-Washington D, Rosenberg RN (2013) Advances in the development of vaccines for Alzheimer's disease. Discov Med 15, 319-326.

[26] Mo J-J, Li J, Yang Z, Liu Z, Feng J-S (2017) Efficacy and safety of anti-amyloid- $\beta$ immunotherapy for Alzheimer's disease: A systematic review and network meta-analysis. Ann Clin Transl Neurol 4, 931-942.

[27] Francis P, Palmer A, Snape M, Wilcock G (1999) The cholinergic hypothesis of Alzheimer's disease: A review of progress. J Neurol Neurosurg Psychiatry 67, 558-558.

[28] McGeer PL, McGeer EG (2013) The amyloid cascadeinflammatory hypothesis of Alzheimer disease: Implications for therapy. Acta Neuropathol (Berl) 126, 479-497.

[29] Sampson EL, Jenagaratnam L, McShane R (2014) Metal protein attenuating compounds for the treatment of Alzheimer's dementia. Cochrane Database Syst Rev, CD005380.

[30] Pisa D, Alonso R, Rábano A, Rodal I, Carrasco L (2015) Different brain regions are infected with fungi in Alzheimer's disease. Sci Rep 5, 15015.

[31] de la Monte SM, Wands JR (2008) Alzheimer's disease is type 3 diabetes-evidence reviewed. J Diabetes Sci Technol 2, 1101-1113.

[32] de la Monte SM (2017) Insulin resistance and neurodegeneration: Progress towards the development of new therapeutics for Alzheimer's disease. Drugs 77, 47-65.

[33] McShane R, Areosa Sastre A, Minakaran N (2006) Memantine for dementia. Cochrane Database Syst Rev, CD003154.

[34] Bush AI, Tanzi RE (2008) Therapeutics for Alzheimer's disease based on the metal hypothesis. Neurotherapeutics 5, 421-432.

[35] Teplan M (2002) Fundamentals of EEG measurement. Meas Sci Rev 2, 11.

[36] Palop JJ, Mucke L (2016) Network abnormalities and interneuron dysfunction in Alzheimer disease. Nat Rev Neurosci 17, 777-792.

[37] Herrmann C, Demiralp T (2005) Human EEG gamma oscillations in neuropsychiatric disorders. Clin Neurophysiol 116, 2719-2733.

[38] Kepecs A, Fishell G (2014) Interneuron cell types are fit to function. Nature 505, 318-326.

[39] Chen C-C, Kiebel SJ, Kilner JM, Ward NS, Stephan KE, Wang W-J, Friston KJ (2012) A dynamic causal 
model for evoked and induced responses. Neuroimage $\mathbf{5 9}$, 340-348.

[40] Tallon-Baudry C, Bertrand O (1999) Oscillatory gamma activity in humans and its role in object representation. Trends Cogn Sci 3, 151-162.

[41] David O, Kilner JM, Friston KJ (2006) Mechanisms of evoked and induced responses in MEG/EEG. Neuroimage 31, 1580-1591.

[42] Lopes da Silva F (2013) EEG and MEG: Relevance to neuroscience. Neuron 80, 1112-1128.

[43] Galambos R, Makeig S, Talmachoff PJ (1981) A 40-Hz auditory potential recorded from the human scalp. Proc Natl Acad Sci U S A 78, 2643-2647.

[44] Pantev C, Roberts LE, Elbert T, Ross B, Wienbruch C (1996) Tonotopic organization of the sources of human auditory steady-state responses. Hear Res 101, 62-74.

[45] Plack CJ, Fay RR, Oxenham AJ, Popper AN (2005) Pitch. Springer New York, New York.

[46] Ross B, Borgmann C, Draganova R, Roberts LE, Pantev C (2000) A high-precision magnetoencephalographic study of human auditory steady-state responses to amplitudemodulated tones. J Acoust Soc Am 108, 679-691.

[47] Pastor MA, Artieda J, Arbizu J, Marti-Climent JM, Peñuelas I, Masdeu JC (2002) Activation of human cerebral and cerebellar cortex by auditory stimulation at 40 Hz. J Neurosci 22, 10501-10506.

[48] Herrmann CS (2001) Human EEG responses to 1-100 Hz flicker: Resonance phenomena in visual cortex and their potential correlation to cognitive phenomena. Exp Brain Res 137, 346-353.

[49] Artieda J, Valencia M, Alegre M, Olaziregi O, Urrestarazu E, Iriarte J (2004) Potentials evoked by chirp-modulated tones: A new technique to evaluate oscillatory activity in the auditory pathway. Clin Neurophysiol 115, 699-709.

[50] Becher A-K, Höhne M, Axmacher N, Chaieb L, Elger CE, Fell J (2015) Intracranial electroencephalography power and phase synchronization changes during monaural and binaural beat stimulation. Eur J Neurosci 41, 254-263.

[51] Kuwada S, Yin TC, Wickesberg RE (1979) Response of cat inferior colliculus neurons to binaural beat stimuli: Possible mechanisms for sound localization. Science 206, 586-588.

[52] Picton TW, John MS, Dimitrijevic A, Purcell D (2003) Human auditory steady-state responses: Respuestas auditivas de estado estable en humanos. Int J Audiol 42, 177-219.

[53] Moore AK, Wehr M (2013) Parvalbumin-expressing inhibitory interneurons in auditory cortex are well-tuned for frequency. J Neurosci 33, 13713-13723.

[54] Fell J, Axmacher N (2011) The role of phase synchronization in memory processes. Nat Rev Neurosci 12, 105-118.

[55] Uhlhaas PJ, Singer W (2006) Neural synchrony in brain disorders: Relevance for cognitive dysfunctions and pathophysiology. Neuron 52, 155-168.

[56] Voicikas A, Niciute I, Ruksenas O, Griskova-Bulanova I (2016) Effect of attention on $40 \mathrm{~Hz}$ auditory steady-state response depends on the stimulation type: Flutter amplitude modulated tones versus clicks. Neurosci Lett 629, 215-220.

[57] Schulze HH (1989) The perception of temporal deviations in isochronic patterns. Percept Psychophys 45, 291-296.

[58] O'Donnell BF, Vohs JL, Krishnan GP, Rass O, Hetrick WP, Morzorati SL (2013) The auditory steady-state response (ASSR): A translational biomarker for schizophrenia. Suppl Clin Neurophysiol 62, 101-112.
[59] McFadden KL, Steinmetz SE, Carroll AM, Simon ST, Wallace A, Rojas DC (2014) Test-retest reliability of the $40 \mathrm{~Hz}$ EEG auditory steady-state response. PLoS One $\mathbf{9}$, e85748.

[60] Light GA, Hsu JL, Hsieh MH, Meyer-Gomes K, Sprock J, Swerdlow NR, Braff DL (2006) Gamma band oscillations reveal neural network cortical coherence dysfunction in schizophrenia patients. Biol Psychiatry 60, 1231-1240.

[61] John MS, Dimitrijevic A, Picton TW (2002) Auditory steady-state responses to exponential modulation envelopes. Ear Hear 23, 106-117.

[62] Rauschecker JP (1998) Cortical processing of complex sounds. Curr Opin Neurobiol 8, 516-521.

[63] Griskova-Bulanova I, Dapsys K, Melynyte S, Voicikas A, Maciulis V, Andruskevicius S, Korostenskaja M (2018) $40 \mathrm{~Hz}$ auditory steady-state response in schizophrenia: Sensitivity to stimulation type (clicks versus flutter amplitude-modulated tones). Neurosci Lett 662, 152-157.

[64] Miyazaki T, Thompson J, Fujioka T, Ross B (2013) Sound envelope encoding in the auditory cortex revealed by neuromagnetic responses in the theta to gamma frequency bands. Brain Res 1506, 64-75.

[65] Lutzenberger W, Pulvermüller F, Elbert T, Birbaumer N (1995) Visual stimulation alters local 40-Hz responses in humans: An EEG-study. Neurosci Lett 183, 39-42.

[66] Lutzenberger W, Pulvermüller F, Birbaumer N (1994) Words and pseudowords elicit distinct patterns of $30-\mathrm{Hz}$ EEG responses in humans. Neurosci Lett 176, 115-118.

[67] Tallon-Baudry C, Bertrand O, Delpuech C, Pernier J (1996) Stimulus specificity of phase-locked and nonphase-locked $40 \mathrm{~Hz}$ visual responses in human. J Neurosci 16, 4240-4249.

[68] Pokorny C, Breitwieser C, Muller-Putz GR (2014) A tactile stimulation device for EEG measurements in clinical use. IEEE Trans Biomed Circuits Syst 8, 305-312.

[69] Muller-Putz GR, Scherer R, Neuper C, Pfurtscheller G (2006) Steady-state somatosensory evoked potentials: Suitable brain signals for brain-computer interfaces? IEEE Trans Neural Syst Rehabil Eng 14, 30-37.

[70] Kübler A, Furdea A, Halder S, Hammer EM, Nijboer F, Kotchoubey B (2009) A Brain-computer interface controlled auditory event-related potential (P300) spelling system for locked-in patients. Ann N Y Acad Sci 1157, 90-100.

[71] Jamali S, Ross B (2014) Sustained changes in somatosensory gamma responses after brief vibrotactile stimulation: Neuroreport 25, 537-541.

[72] C-2 Tactors. https://www.eaiinfo.com/product/c2/, Accessed on March 22, 2018.

[73] Giabbiconi C-M, Trujillo-Barreto NJ, Gruber T, Müller MM (2007) Sustained spatial attention to vibration is mediated in primary somatosensory cortex. Neuroimage 35, 255-262.

[74] Severens M, Farquhar J, Desain P, Duysens J, Gielen C (2010) Transient and steady-state responses to mechanical stimulation of different fingers reveal interactions based on lateral inhibition. Clin Neurophysiol 121, 2090-2096.

[75] Kandel ER (2013) Principles of neural science. McGrawHill, New York.

[76] Herrmann CS, Rach S, Neuling T, Strüber D (2013) Transcranial alternating current stimulation: A review of the underlying mechanisms and modulation of cognitive processes. Front Hum Neurosci 7, 279.

[77] Kanai R, Paulus W, Walsh V (2010) Transcranial alternating current stimulation (tACS) modulates cortical 
excitability as assessed by TMS-induced phosphene thresholds. Clin Neurophysiol 121, 1551-1554.

[78] Laczó B, Antal A, Niebergall R, Treue S, Paulus W (2012) Transcranial alternating stimulation in a high gamma frequency range applied over V1 improves contrast perception but does not modulate spatial attention. Brain Stimulat 5, 484-491.

[79] Strüber D, Rach S, Trautmann-Lengsfeld SA, Engel AK, Herrmann CS (2014) Antiphasic $40 \mathrm{~Hz}$ oscillatory current stimulation affects bistable motion perception. Brain Topogr 27, 158-171.

[80] Marshall L, Helgadóttir H, Mölle M, Born J (2006) Boosting slow oscillations during sleep potentiates memory. Nature 444, 610-613.

[81] Kirov R, Weiss C, Siebner HR, Born J, Marshall L (2009) Slow oscillation electrical brain stimulation during waking promotes EEG theta activity and memory encoding. Proc Natl Acad Sci U S A 106, 15460-15465.

[82] Ross B, Herdman AT, Pantev C (2005) Stimulus induced desynchronization of human auditory $40-\mathrm{Hz}$ steady-state responses. J Neurophysiol 94, 4082-4093.

[83] DeLosAngeles D (2010) Eletroencephalographic, Cognitive and Autonomic Correlates of States of Concentrative Meditation. Flinders University, Adelaide, South Australia.

[84] Kösem A, Gramfort A, van Wassenhove V (2014) Encoding of event timing in the phase of neural oscillations. Neuroimage 92, 274-284.

[85] Fitzgibbon SP, DeLosAngeles D, Lewis TW, Powers DMW, Grummett TS, Whitham EM, Ward LM, Willoughby JO, Pope KJ (2016) Automatic determination of EMG-contaminated components and validation of independent component analysis using EEG during pharmacologic paralysis. Clin Neurophysiol 127, 1781-1793.

[86] Bertrand O, Tallon-Baudry C (2000) Oscillatory gamma activity in humans: A possible role for object representation. Int J Psychophysiol 38, 211-223.

[87] Buzsáki G (2006) Rhythms of the brain. Oxford University Press, Oxford; New York.

[88] Goncharova I, McFarland D, Vaughan T, Wolpaw J (2003) EMG contamination of EEG: Spectral and topographical characteristics. Clin Neurophysiol 114, 1580-1593.

[89] Mirzadeh Z, Bari A, Lozano AM (2016) The rationale for deep brain stimulation in Alzheimer's disease. J Neural Transm 123, 775-783.

[90] Fraser C, Power M, Hamdy S, Rothwell J, Hobday D, Hollander I, Tyrell P, Hobson A, Williams S, Thompson $\mathrm{D}$ (2002) Driving plasticity in human adult motor cortex is associated with improved motor function after brain injury. Neuron 34, 831-840.

[91] Silva VF da, Ribeiro AP, Santos VA dos, Nardi AE, King ALS, Calomeni MR (2015) Stimulation by light and sound: Therapeutics effects in humans. Systematic Review. Clin Pract Epidemiol Ment Health 11, 150-154.

[92] Vieira M (2008) Efeitos de um programa conjugado da metodologia de luz e som e prática motriz complexa em funções de memória em indivíduos portadores da doença de Alzheimer: Relações com hemisfericidade. Universidade Castelo Branco, Rio de Janeiro, Brazil.

[93] Clements-Cortes A, Ahonen H, Evans M, Freedman M, Bartel L (2016) Short-term effects of rhythmic sensory stimulation in Alzheimer's disease: An exploratory pilot study. J Alzheimers Dis 52, 651-660.

[94] Ribary U, Ioannides AA, Singh KD, Hasson R, Bolton JP, Lado F, Mogilner A, Llinás R (1991) Magnetic field tomography of coherent thalamocortical $40-\mathrm{Hz}$ oscillations in humans. Proc Natl Acad Sci U S A 88, 11037-11041.

[95] Llinas R, Ribary U (1993) Coherent 40-Hz oscillation characterizes dream state in humans. Proc Natl Acad Sci U S A 90, 2078-2081.

[96] Next Wave Headquarters, Next Wave Physioacoustic MX therapy chair. http://www.nextwaveworldwide.com/ $\% 20$ products/physioacoustic-mx-therapy-chair/, Accessed on March 24, 2018.

[97] Tariq SH, Tumosa N, Chibnall JT, Perry MH, Morley JE (2006) Comparison of the Saint Louis University Mental Status Examination and the Mini-Mental State Examination for detecting dementia and mild neurocognitive disorder-a pilot study. Am J Geriatr Psychiatry 14, 900910.

[98] Clements-Cortes A, Bartel L, Ahonen H, Freedman M, Evans M, Tang-Wai DF (2017) Can rhythmic sensory stimulation decrease cognitive decline in Alzheimer's disease?: A clinical case study. Music Med $\mathbf{9}$, 174-177.

[99] Clements-Cortes A, Bartel L, Ahonen H, Freedman M (2017) The potential of rhythmic sensory stimulation treatments for persons with Alzheimer's disease. Music Med 9, 167-173.

[100] Punkanen M, Ala-Ruona E (2012) Contemporary vibroacoustic therapy: Perspectives on clinical practice, research, and training. Music Med 4, 128-135.

[101] Bartel L, Chen R, Alain C, Ross B (2017) Vibroacoustic stimulation and brain oscillation: From basic research to clinical application. Music Med 9, 153-166.

[102] Naro A, Corallo F, De Salvo S, Marra A, Di Lorenzo G, Muscará N, Russo M, Marino S, De Luca R, Bramanti P, Calabrò RS (2016) Promising role of neuromodulation in predicting the progression of mild cognitive impairment to dementia. J Alzheimers Dis 53, 1375-1388.

[103] Hugo J, Ganguli M (2014) Dementia and cognitive impairment. Clin Geriatr Med 30, 421-442.

[104] Calomeni MR, Furtado da Silva V, Velasques BB, Feijó OG, Bittencourt JM, Ribeiro de Souza e Silva AP (2017) Modulatory effect of association of brain stimulation by light and binaural beats in specific brain waves. Clin Pract Epidemiol Ment Health 13, 134-144.

[105] Başar E, Femir B, Emek-Savaş DD, Güntekin B, Yener GG (2017) Increased long distance event-related gamma band connectivity in Alzheimer's disease. Neuroimage Clin 14, 580-590.

[106] Rossini PM, Del Percio C, Pasqualetti P, Cassetta E, Binetti G, Dal Forno G, Ferreri F, Frisoni G, Chiovenda P, Miniussi C, Parisi L, Tombini M, Vecchio F, Babiloni C (2006) Conversion from mild cognitive impairment to Alzheimer's disease is predicted by sources and coherence of brain electroencephalography rhythms. Neuroscience 143, 793-803.

[107] van Deursen JA, Vuurman EFPM, van KranenMastenbroek VHJM, Verhey FRJ, Riedel WJ (2011) $40-\mathrm{Hz}$ steady state response in Alzheimer's disease and mild cognitive impairment. Neurobiol Aging 32, 24-30.

[108] Wilson C (2018) Brainwave therapy for Alzheimer's. Can flickering lights, low hums and vibrating pads zap brain plaques. New Sci 237, 6.

[109] CLS (Coalition for the Life Sciences), Shining a Light on the Treatment of Alzheimer's Disease. https://www. coalitionforlifesciences.org/shining-light-treatmentalzheimers-disease/, Accessed on June 5, 2018. 
[110] Neuroscience 2017, November 11-15, Washington, DC, Society for Neuroscience, Advancing the Understanding of the Brain and Nervous System, Last updated April 1, 2018, Accessed on April 1, 2018.

[111] Cognito Therapeutics. https://www.cognitotx.com/, Accessed on April 1, 2018.

[112] Freund H-J, Kuhn J, Lenartz D, Mai JK, Schnell T, Klosterkoetter J, Sturm V (2009) Cognitive functions in a patient with Parkinson-Dementia syndrome undergoing deep brain stimulation. Arch Neurol 66, 781-785.

[113] Kuhn J, Hardenacke K, Lenartz D, Gruendler T, Ullsperger M, Bartsch C, Mai JK, Zilles K, Bauer A, Matusch A, Schulz R-J, Noreik M, Bührle CP, Maintz D, Woopen C, Häussermann P, Hellmich M, Klosterkötter J, Wiltfang J, Maarouf M, Freund H-J, Sturm V (2015) Deep brain stimulation of the nucleus basalis of Meynert in Alzheimer's dementia. Mol Psychiatry 20, 353-360.

[114] Hamani C, Stone SS, Garten A, Lozano AM, Winocur G (2011) Memory rescue and enhanced neurogenesis following electrical stimulation of the anterior thalamus in rats treated with corticosterone. Exp Neurol 232, 100-104.

[115] Laxton AW, Tang-Wai DF, McAndrews MP, Zumsteg D, Wennberg R, Keren R, Wherrett J, Naglie G, Hamani C, Smith GS, Lozano AM (2010) A phase I trial of deep brain stimulation of memory circuits in Alzheimer's disease. Ann Neurol 68, 521-534.

[116] Sankar T, Chakravarty MM, Bescos A, Lara M, Obuchi T, Laxton AW, McAndrews MP, Tang-Wai DF, Workman CI, Smith GS, Lozano AM (2015) Deep brain stimulation influences brain structure in Alzheimer's disease. Brain Stimulat 8, 645-654.
[117] Solito E, Sastre M (2012) Microglia function in Alzheimer's disease. Front Pharmacol 3, 14.

[118] Striano S, Coppola A, del Gaudio L, Striano P (2012) Reflex seizures and reflex epilepsies: Old models for understanding mechanisms of epileptogenesis. Epilepsy Res 100, 1-11.

[119] Stam CJ, van Cappellen van Walsum AM, Pijnenburg YAL, Berendse HW, de Munck JC, Scheltens P, van Dijk BW (2002) Generalized synchronization of MEG recordings in Alzheimer's disease: Evidence for involvement of the gamma band. J Clin Neurophysiol 19, 562-574.

[120] Koenig T, Prichep L, Dierks T, Hubl D, Wahlund LO, John ER, Jelic V (2005) Decreased EEG synchronization in Alzheimer's disease and mild cognitive impairment. Neurobiol Aging 26, 165-171.

[121] Stam CJ, Jones BF, Manshanden I, van Cappellen van Walsum AM, Montez T, Verbunt JPA, de Munck JC, van Dijk BW, Berendse HW, Scheltens P (2006) Magnetoencephalographic evaluation of resting-state functional connectivity in Alzheimer's disease. Neuroimage 32, 1335-1344.

[122] Wang J, Fang Y, Wang X, Yang H, Yu X, Wang H (2017) Enhanced gamma activity and cross-frequency interaction of resting-state electroencephalographic oscillations in patients with Alzheimer's disease. Front Aging Neurosci 9, 243.

[123] van Deursen JA, Vuurman EFPM, Verhey FRJ, van Kranen-Mastenbroek VHJM, Riedel WJ (2008) Increased EEG gamma band activity in Alzheimer's disease and mild cognitive impairment. J Neural Transm 115, 1301-1311. 